\title{
Mūsā Cālīnūs' Treatise on the Natures of Medicines and Their Use
}

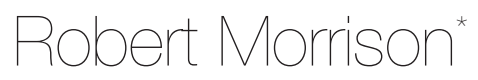

\begin{abstract}
This article introduces and presents a transcription and annotated translation of a medical text in Ottoman Turkish authored by Mūsā Cālīnūs (d. after 1542). The treatise is entitled Risāla fi Ṭabä'i ' al-adviya va-isti'mäliha (Treatise on the Natures of Medicines and Their Use). This article analyses the degrees of the qualities (e.g., heat, cold, wetness, and dryness) of various materia medica and how, on that basis, certain drugs affect, effect, and preserve health. There are three reasons why this brief, seemingly pedestrian text merits more extensive study. First, it refers to the medieval Latin physicians Bernard de Gordon (fl. 12701330) and Arnaldo di Villanova (1234-1310) for perhaps the first time in Turkish (and Islamic) literature. Second, Mūsā Câlinnūs must have believed that there was an audience at Beyazid II's court for the contents of medical texts composed in Latin. Third, as Mūsā Câlīnūs is a probable conduit through which information about the astronomy of Islamic societies could have reached the Veneto around 1500, his interest in the contents of Latin medical texts meant that he was a scholarly intermediary who carried information in at least two directions.
\end{abstract}

Keywords: Beyazid II, medicine, Arnaldo di Villanova, pharmacology, al-Kindī, Ibn Rushd.

* Prof., Bowdoin College, Religion Department.

Correspondence: rgmorris@bowdoin.edu 
$\mathrm{T}$ his article introduces and presents a transcription and annotated translation of the Risāla fi Țaba'i' al-adviya va-isti'mālihā (Treatise on the Natures of Medicines and Their Use $)^{1}$, a medical text in Ottoman Turkish authored by Mūsā Cālīnūs (d. after 1542). Mūsā Cālīnūs wrote also in Arabic and Hebrew (as Moses Galeano) and produced an Arabic version of the Latin canons of the Almanach Perpetuum. ${ }^{2}$ This text, which exists in I.U. Y1ld1z Tip 352 (also numbered IU Yildız Tip 7120)3 ${ }^{3}$, is his only known composition in Ottoman Turkish and, according to him, was commissioned by Ahi Çelebi, Beyazid II's (r. 1481-1512) chief physician. ${ }^{4}$ On folio $2 \mathrm{a}$, the text refers to Ahi Çelebi as the chief of physicians, ra'ìs al-hukamā. If we take this phrase to be synonymous with hekimbaşı, then the text must have been written in 1507 or thereafter, as that was when Ahi Çelebi was first appointed chief physician under Beyazid II. ${ }^{5}$ In addition to his original compositions, Ahi Çelebi translated Ibn al-Nafīs' al-Mūcaz fī al-țibb into Turkish and authored a commentary on Çaġmīnī's Känūnça. ${ }^{6}$ Because Osmanlı Tıbbi Bilimler Literatürü Tarihi ${ }^{7}$ attributed the treatise to Mūsā b. Hāmūn al-Isrā’ìlì, Mūsā Cālīnūs was not mentioned in that modern bio-bibliographical work. Work on Mūsā Cālīnūs' (which he wrote as Moses Galeano) Puzzles of Wisdom (Heb. Ta'alumot hok uncovered accounts of intrigue and competition among physicians at the sultan's

Cevat İzgi, Osmanlı Medreselerinde İlim, (Istanbul: İz Yayıncllı, 1997): vol. 2 of 2, 23.

On the Arabic version of the canons of the Almanach Perpetuum, see Julio Samsó: "Abraham Zacut and Jose' Vizinho's Almanach Perpetuum in Arabic (16th-19th C.)," Centaurus XLVI, (2004): 82-97, at 83.

The unique MS is has a copying date of $966 \mathrm{~A}$.H. and has some marginalia that include fi ba'ż al-nusah indicating that the copyist or a reader had access to multiple MSS of the text

Ahi Çelebi was Muhammad b. Kamāl al-Tabrīzi. See İzgi, Osmanlı Medreselerinde İlim, vol. 2 of 2, 23.

Ekmeleddin İhsanoglu (ed.), Osmanlı Trbbi Bilimler Literatürü Tarihi (Istanbul: Ircica, 2008), vol. 1 of 4, 105. We know that Mūsā Cälīnūs' translation of the Latin canons to the Almanach Perpetuum was dedicated to the Kãzī 'Abd al-Rahmān Mu'ayyadzādah. Because Mu'ayyadzädah was appointed to the position of käżi-'askar of Rumelia in 1505 and of Anatolia in 1501, we have a terminus post quem for that translation, a date that meshes well with the time frame for the composition of the present text. See Judith Pfeiffer: “Teaching the Learned: Jalāl al-Dīn al-Dawānī's Ijāza to Mu'ayyadzāda 'Abd al-Rahmān Efendi and the Circulation of Knowledge between Fārs and the Ottoman Empire at the Turn of the Sixteenth Century," in Maurice Pomerantz and Aram Shahin (eds.), The Heritage of Arabo-Islamic Learning, (Leiden and Boston: Brill, 2016): 284-332, at 287.

İhsanoğlu, Osmanlı Tıbbi Bilimler, vol. 1, 108-9. On the Känūnca and the date of its composition by a Cağminnī, see Sally Ragep, Mahmūd ibn Muhammad ibn 'Umar al-Jaghminin's al-Mulakhkha fi al-hay'a albasita: An Edition, Translation, and Study, (Ph.D. Dissertation, McGill University, 2014): 18-41.

For some information about the text, see İhsanoğlu, Osmanl Trbbi Bilimler, vol. 1, 135. This book attributed the text to Mūsā b. Hāmūn al-Isräîlì (d. 1554). I disagree with this attribution because no patronymic (b. Hāmūn) is given in the treatise, because Mūsā Cālinnūs referred to himself as al-Isrầilī in his Arabic treatise on homocentric astronomy and because the present text's strong statement about the impossibility of the epicycle and eccentric meshes with everything Mūsā Cālinnūs said about astronomy. Moreover, İzgi (Osmanlı Medreselerinde İlim, vol. 2, 23) identified the author as 'Mūsā Cālinuũs b. Yehüda al-Tabīb', whom I would take to be Moses b. Judah Galeano (a.k.a. Mūsā Cālīnūs). 
court. ${ }^{8}$ There is other evidence of an interest in his work at that court as well, for an Arabic text he wrote on astronomy ended up in Ahmet III's collection at the Topkap1 Library. ${ }^{9}$ That treatise includes a long passage from a Judeo-Arabic text, The Light of the World, that Mūsā Cālinnūs brought to the attention of scholars in the Veneto. ${ }^{10}$

Risāla fi țaba' 'i' al-adviya va-isti 'mālihā analyses the degrees of the qualities (e.g., heat, cold, wetness, and dryness) of various materia medica and how, on that basis, certain drugs affect, effect, and preserve health. This brief, seemingly pedestrian text merits more extensive study because, first of all, it refers to the medieval Latin physicians Bernard de Gordon (fl. 1270-1330) and Arnaldo di Villanova (12341310) for perhaps the first time in Turkish literature. ${ }^{11}$ These are significant references, for while these physicians' Latin texts drew upon the medicine of Islamic societies, the Latin texts themselves were not translated into Arabic, Persian, or Turkish. While we know that later Ottoman intellectuals took an interest in the intellectual life of Europe, the present text is evidence of an earlier interest in Latin medical texts. ${ }^{12}$

Second, the treatise's first page states that Mūsā Cālīnūs, while composing the treatise, would draw upon "the words of Islamic, Frankish, Greek, and Jewish physicians." ${ }^{13}$ He therefore must have believed that there was an audience at Beyazid II's court for the contents of medical texts composed in Latin (as well as in Hebrew). Previous scholarship on Mūsā Cālīnūs has shown that he travelled to

On these accounts, see See Y. Tzvi Langermann: "A Compendium of Renaissance Science: Ta'alumot hokkmah by Moshe Galeano," Aleph: Historical Studies in Science and Judaism VII, (2007): 283-318 and Langermann: "Medicine, Mechanics and Magic from Moses ben Judah Galeano's Ta'alumot hoknma," Aleph: Historical Studies in Science and Judaism IX, (2009): 353-77.

9 Robert Morrison: “An Astronomical Treatise by Mūsā Jālīnūs alias Moses Galeano," Aleph: Historical Studies in Science and Judaism X/2, (2011): 315-53, at 385-6. Both the astronomy text and the medical text opposed Ptolemaic theoretical astronomy and favored an approach that rejected epicycles and eccentrics.

10 On Mūsā Cālinnūs' role in this episode of scholarly exchange, see Ibn Nahmias, Joseph, (ed., trans. and comm.) Robert Morrison, The Light of the World: Astronomy in al-Andalus, (Berkeley and London: The University of California Press, 2016): 41-4.

11 Abdülhak Adnan-Adıvar, Osmanlı Türklerinde İlim, (Istanbul: Maarif Matbaası, 1943): 54.

12 On Ottoman interest in European science from a slightly later period, see Feza Günergün: "Ottoman Encounters with European science: sixteenth and seventeenth-century translations into Turkish," in P. Burke and R. Po-chia Hsia (eds.), Cultural Translation in Early Modern Europe, (Cambridge: Cambridge University Press, 2007): 192-211. On the enduring interest of physicians who wrote in Latin in Arabic pharmacopeia, even after Jalīnūs' career, see Teresa Huguet-Termes, “Islamic Pharmacology and Pharmacy in the Latin West: An Approach to Early Pharmacopeias," European Review XVI/2, (2008): 229-39. fol. $1 b$. 
the Veneto and had contact with Christian scholars there. ${ }^{14}$ Thus, he is a probable conduit through which information about the astronomy of Islamic societies could have reached the Veneto around $1500 .{ }^{15}$ His interest in the contents of Latin medical texts is more evidence that he was a scholarly intermediary who carried information in at least two directions. ${ }^{16}$ Mūsā Cālinnūs was not the only scholarly intermediary in his family. ${ }^{17}$ The article will explain that it is quite possible that his information about Latin medical texts came, at least in part, from Joshua Lorci's (d. 1419) Gerem ha-ma'alot, a Hebrew text composed originally in Arabic, that Mūsā Câlīnūs cited in his Puzzles of Wisdom. ${ }^{18}$ Third, the present treatise enhances our knowledge of the state of the theory behind pharmaceutical practice during the time of Beyazid II, particularly as to how the rankings of drugs' qualities, introduced into the medicine of Islamic societies by al-Kindī (d. 873), were discussed and modified over time. Indeed, recent research has shown that scholars in Islamic societies continued to work on pharmacological theory after Ibn Rushd. ${ }^{19}$

The current treatise participates in a debate that began with the appropriation of Galen's pharmacology in Kindî's On Degrees. ${ }^{20}$ Galen had postulated that drugs were hot, cold, dry, and moist to varying degrees of intensity, as well as in a state of equilibrium (subsequently expressed in Arabic as $i^{t} t i d \bar{a} l$ ). ${ }^{21}$ The degrees of hot, cold, wet, and dry in simple medicines determine how hot/cold/dry/wet a non-equili-

14 See Langermann, “A Compendium"; Langermann, "Medicine, Mechanics and Magic"; Robert Morrison, "A Scholarly Intermediary Between the Ottoman Empire and Renaissance Europe," Isis CV, (2014): 32-57; and Morrison, "An Astronomical Treatise."

15 Morrison, “A Scholarly Intermediary”; see also Langermann, “A Compendium,” 290-5.

16 Samsó ("Abraham Zacut”) has already brought to light Mūsā Cālinnūs' translation of the Latin (or perhaps Castilian) canons of the Almanach Perpetuum into Arabic.

17 On an older contemporary of Mūsā Cālīnūs, named Moses Galeano, see Robert Morrison, "The Role of Oral Transmission for Astronomy Among Romaniot Jews," in Y. Tzvi Langermann and Robert Morrison (eds), Texts in Transit, (University Park, PA: Pennsylvania State University Press, 2016): 1028. See 15 for a brief mention of Risāla fi Tabä'i' al-adviya.

18 On Lorci, see Beinart, Haim. "Lorki (i.e., of Lorca), Joshua." Encyclopaedia Judaica, ed. Michael Berenbaum and Fred Skolnik. 2nd ed. Vol. 13. Detroit: Macmillan Reference USA, 2007. 192-193. Gale Virtual Reference Library. Web. 4 Sept. 2016.

19 See, e.g., Danielle Jacquart, "Islamic Pharmacology in the Middle Ages: Theories and Substances," European Review XVI, 2 (2008): 219-27 and Leigh Chipman, The World of Pharmacy and Pharmacists in Mamlūk Cairo, (Boston: Brill, 2010).

20 Langermann has noted ("Another Andalusian Revolt," 352) that the relatively few medieval authors interested in the degrees of compound medicines were not too concerned with the question of how to interpret Galen's writings on the subject.

21 Nutton, Vivian (London). “Galen of Pergamum.” Brill's New Pauly. Antiquity volumes edited by Hubert Cancik and Helmuth Schneider. Brill Online, 2016. See also Peter Adamson, al-Kindī (Oxford University Press, 2006): 162-3. 
brate compound medicine is. Galen was less committed than Kindī to quantifying the degrees of these qualities' intensity. ${ }^{22}$ Kindī, however, drew several conclusions about the relationship of something in equilibrium to something in the first degree. First, he determined the relationships between the degrees mathematically, not through observation. Second, he decided that this relationship was doubling $(2,4,8,16)$, as opposed to progressing by integers $(1,2,3,4) .{ }^{23}$ For him, "something hot in the fourth degree is 8 times as something hot in the first degree and 16 times as hot as an equilibrated compound." 24

These conclusions became the starting point for subsequent discussions among physicians of Islamic societies. Most important, in his Kulliyyāt, Ibn Rushd (d. 1198; Latin: Averroës) argued that the more appropriate ratio between degrees would be that of the integers. ${ }^{25}$ We find his most developed views on the subject in his treatise on theriac (an antidote to poison). ${ }^{26}$ Ibn Rushd criticized Kindi's pharmacology in terms of the extent to which the computus of the degrees accorded with experience. Kindi explained that a drug with the first degree of a quality was the minimum amount necessary to produce a given effect in the person who ingested it. ${ }^{27} \mathrm{He}$ did not assert that one would be able to somehow perceive eight times the quality in a compound that has that quality in the fourth degree. Although Kindī would use experiment and observation to argue for his system of doubling and against other systems, experiment and observation were not the foundation of his theories of degrees. Rather, the mathematical relation of doubling was the most natural. ${ }^{28}$

Following the translation of Arabic scientific and philosophic texts into Latin, scholars writing in Latin engaged in this debate about the computus of simple

Galen (trans. P.N. Singer), Selected Works (Oxford University Press, 1997), 248 (Kühn 608). But see Singer's introduction, xiii (referring to Galen's treatises on the composition of medicines which are accessible through Kühn's edition).

23 Adamson, al-Kindī, 162-4.

24 Adamson, al-Kindī, 164.

25 Kindi had argued that with an integer progression $(1,2,3,4)$, the problem was that the ratio between the integers was, in fact, constantly changing as 3:2 is not equal to 2:1. For a study of Ibn Rushd's critiques of al-Kindī, see Y. Tzvi Langermann: "Another Andalusian Revolt? Ibn Rushd's Critique of alKindi's Pharmacological Computus," in Jan P. Hogendijk and A.I. Sabra (eds.), The Enterprise of Science in Islam: New Perspectives, (Cambridge and London: MIT Press, 2003): 351-372.

Langermann, "Another Andalusian Revolt," 353. The treatise on theriac (Kitāb al-tiryāq) is in Ibn Rushd (Jūrj Qanawātī and Sa'īd Zā’id eds.), Rasā’il Ibn Rushd al-țibbiyya (Cairo: al-Hay’a al-Mișriyya al-'āmma li-l-kitāb, 1987): 387-422. 
and compound medicines. Arnaldo di Villanova produced a Latin text based on Kindī's De Gradibus. Michael McVaugh found Arnaldo's theory of degrees to be novel and, as such, sufficiently independent of the Arabic authorities respected at Montpellier where Arnaldo worked. ${ }^{29}$ To address the question of the degree of a compound medicine, Arnaldo determined that " $[\mathrm{t}]$ he intensive degree of a compound formed from simples hot in the second degree and cold in the first could be calculated as follows: $2^{\circ}$ hot $4: 1 ; 1^{\circ}$ cold $1: 2$; [the total is] $5: 3$ and would thus be just less than the first degree of hotness." ${ }^{30}$ That same question attracted Mūsā Cālīnūs' attention, as did that of how and whether the drug's effect varies with respect to the amount given. ${ }^{31}$

Bernard di Gordon's own treatise De Gradibus, completed in 1303, defended and presented Arnaldo's methods in a more accessible manner and tried to outline an experimental foundation to support the mathematical theory. ${ }^{32}$ While Mūsā Cālīnūs mentioned Arnaldo twice on his own, as well as Bernard once on his own, there was a place where he referred to both of them together. ${ }^{33}$ He maintained that Bernard agreed with Kindi that the ratio of one degree of a quality to the next was that of doubling. ${ }^{34}$ Just as Kindī saw mathematics as an underlying, fundamental truth, ${ }^{35}$ Bernard wrote that "we cannot know without numbers." 36 While the ratio of doubling might not hold up well in an experiment, Mūsā Cālīnūs claimed that Arnaldo and Bernard believed doubling to be the truth because it yielded the correct mathematical ratios. ${ }^{37}$ The attraction of this ratio must have been its application to the production of compound medicines, although Bernard ended up using it as a way to describe medicines that were already in use. ${ }^{38}$ Moreover, a medicine could contain quantities (or measures) of Bulletin of the History of Medicine XLIII, (1969): 397-413, at 398.

30 McVaugh, "Quantified," 401. These solutions drew on Averroës and al-Kindī's work.

31 McVaugh, "Quantified," 400.

32 Luke E. Demaitre, Doctor Bernard de Gordon: Professor and Practitioner (Toronto: Pontifical Institute of Mediaeval Studies, 1980): 20 and 50. A recent publication on Bernard De Gordon's commitment to medical astrology is Danielle Jacquart: "Bernard de Gordon et l'astrologie," Centaurus XLV, (2003): 151-8. fol. 4a. "Therefore Arnaldo and Gordon worked according to this..."

34 fol. 3b. See also McVaugh, "Quantified," 401 on Arnaldo's acceptance of the ratio of doubling.

35 Adamson, al-Kindī, 165.

36 Demaitre, Doctor Bernhard, 20.

37 fol. 4 a.

38 McVaugh, "Quantified," 405. 
both hot and cold, which would increase or decrease depending upon how much of the medicine was administered. ${ }^{39}$ The medicine's overall degree would not be affected by the amount administered, meaning that the practical and theoretical approaches could not be fully reconciled.

Here is an outline of the text:

Chapter One

The reasons for making a compound medicine, communicated in a tree diagram

Chapter Two

Some preliminaries necessary for knowing a compound's rank

First preliminary: How to determine a compound's rank

Second preliminary: As the quantities increase, so do the qualities

Third preliminary: A medicine's degree cannot be known solely on the basis of its effect; one must know the degree's measure as well

Fourth preliminary: The parts of a quality at each degree

Fifth preliminary: How medicines affect each other when combined

\section{Chapter Three}

Recipes that specify the qualities of the constituent simple medicines and the simples' quantities

Chapter Four

What to add to make a compound medicine return to equilibrium

Chapter Five

Question one: Can compound medicines have opposing effects on the secondary properties? 
Question two: How does one predict the effect of the combination of medicines of a certain rank?

Question three: What is the ratio of one degree to the next?

Question four: How do medicines of different degrees affect each other?

Question five: How do medicines affect each other when mixed?

This article contains a minimally corrected transcription as well as a more heavily annotated translation of Mūsā Cālīnūs' Risāla fi țabā'i ' al-adviya va-isti'mālihā. The MS indicates the Ottoman "g" with a "k" and, generally, the "ç" with a "j." When the MS has lengthy quotations in Arabic, I have transcribed those sections according to standard Arabic orthography. 


$$
\text { بسم الله الرحمن الرحيم }
$$

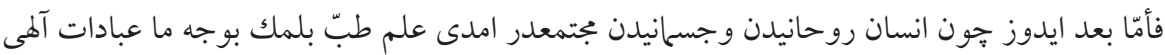

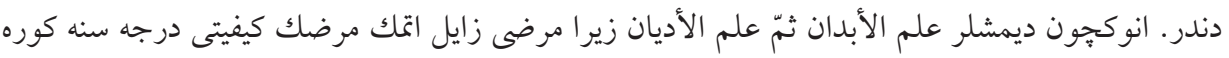

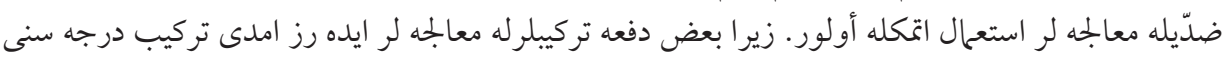

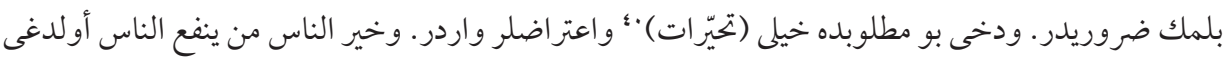

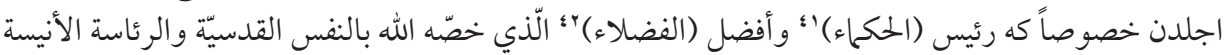

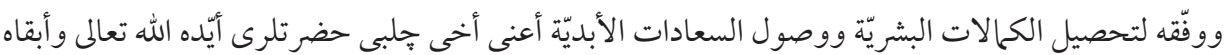

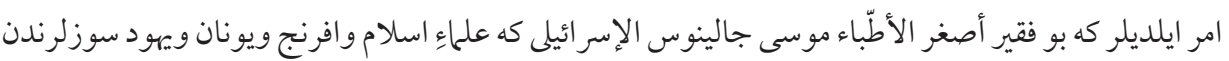
مفيد وخختصر ومفسّر رساله جمع ايدم. فأمّا مرضلرك كيفيّلرى درجه لرى وهر بر أدويه قدرى يعنى نه مقدار استعال اتمكله درجه سى اثرى

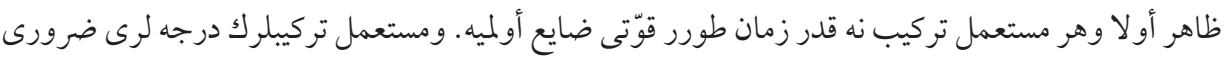
كتابمده ذكر ايدم ان شاء الله الرحمن. الفصل الأوّل تركيب اتدو كمز سببلرى تقسيم عقليله بلدرر زيرا مر اد تركيب درجه سنى بلدرمك در . وأطبّا قاعده سى بودر كه بسيط أدويه كافى أولدغى وقتن تركيب اتميه وز. امدى تركيب اتدوكمز سببلرى بلدئ بلدرمكدر

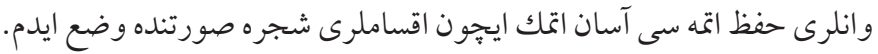

$$
\text { ادويه مفرده }
$$$$
\text { ا. يا معالجه يه دخلى اوله }
$$

|ll

ي $\quad 40$

غاتئ : In the margin

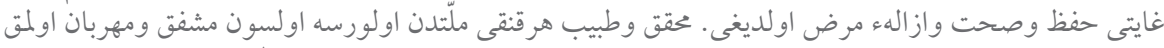

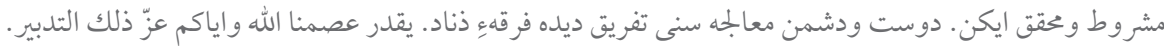




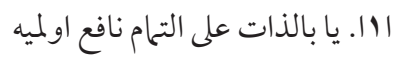

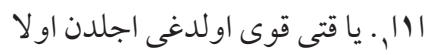

|||,|, يا قوتلرى :جوق اولدقلرى اجلدن اوله ومقصود دكلردر

|' |', . ياخود كيم مقصود اولان نسنه ده قتى قوى اولدغى اجلدن اوله انى أضعف اتمسنه تركيبه محتاج اوله يالكوز مقصو دمزه بالذات نافع دكلدر

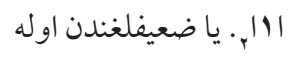

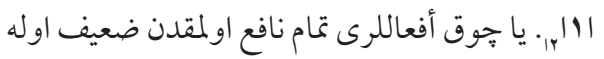

| Iار|, واول أفعال يا مشابه او لالر نته كيم ترياق فعلى جميع اغولره نافع اولمسنه مركب اولمشدر

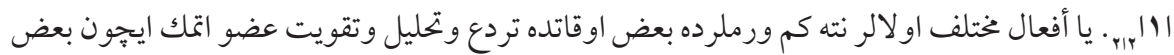

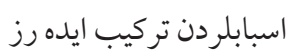

$$
\text { |'اب.ب. ياخود بر يالكوز فعله دخى ضعيف اولا }
$$

|'اب ب ـ يا حدّ ذاتنده ضعيف اوله معاون نسنه يه محتاج اوله ملح اندرانى غاريقوه نه وزنجبيل تربده قاتدغمز

|'|"ابץ. ياخود كيم اكلندكى ويا قتى سريع اولدغى اجلدن اوله اكلندرمكيجون غيرى نسنه قتارز نته كم

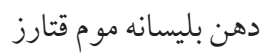

|"اببr. وياخود مقصود اولان يره وارمقدن ضعيف اوله نته كم افعى ترياقده قاترز ساير أدويه لرى أعضاء

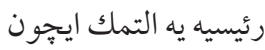

$$
\text { الب. بابالعرض }
$$

الب ا. ياخود كيم كركلو أعنى دوسته مضرّ اولدغى أجلدن اوله بو بنجلين إصلاح ايجِون تركبه محتاج در

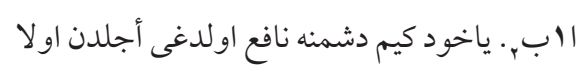

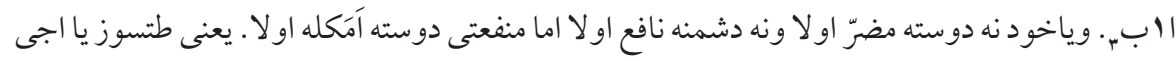
اولماغين انوك دفعيجون تركيبه محتاج در ابץ ـ يا على التهام مقصوده يرايه وبونك بكى يه تركيب اتكك حاجت دكولدر

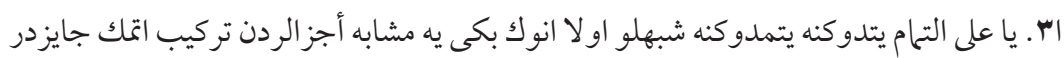
ب. يا اولميه واكر دخلى يا مناسبتى يوغسه قطعاً مقصو د دكلدر 
الفصل الثانى كه تركيك مرتبه سنى بلمسنه بعض ضرورى مقدمه لر ذكر ايدر. أوّلكى مقدمه بو در كه

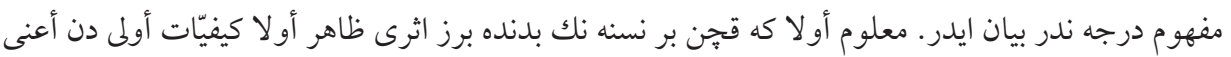

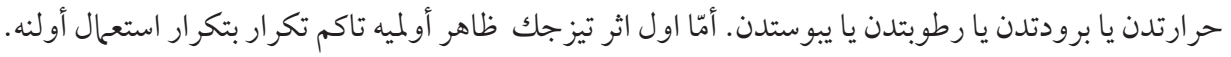

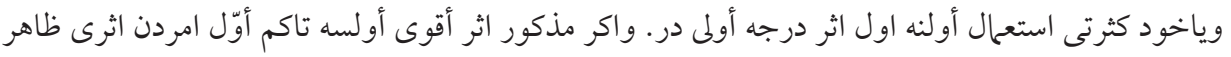

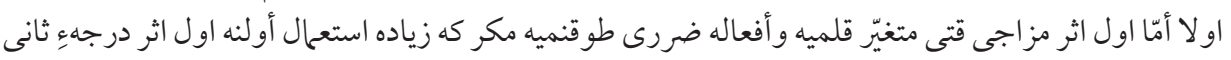

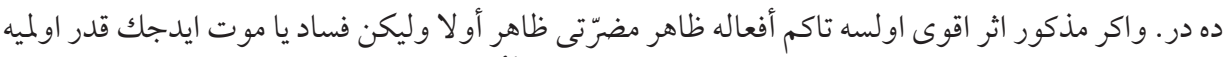

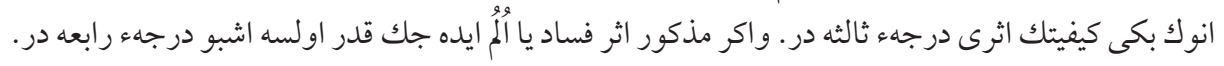
اكنجى مقدّمه بودر كم كميّّلر جهوغالنجه ومضاعف اولدغنجه كيفيّاتلرى دخى اكا كوره آرتار . مثلاً صندل

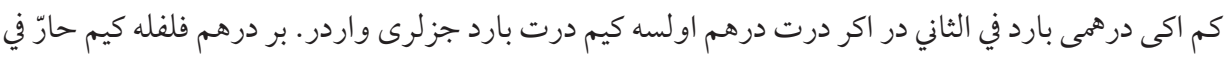

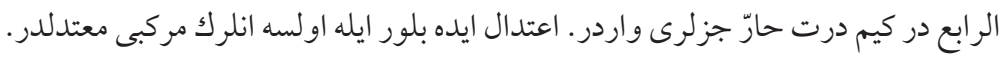

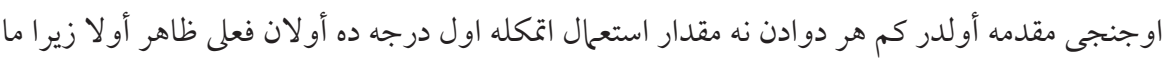

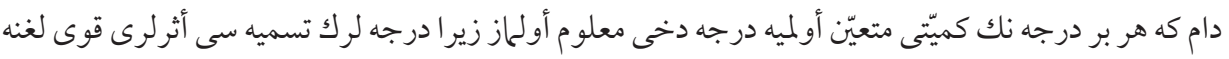

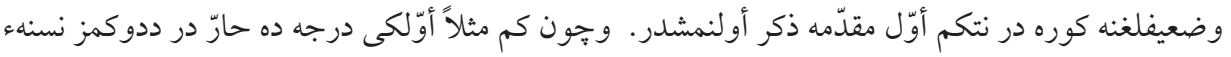

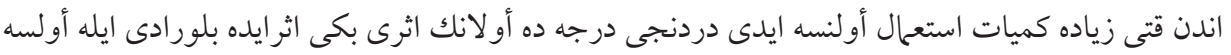

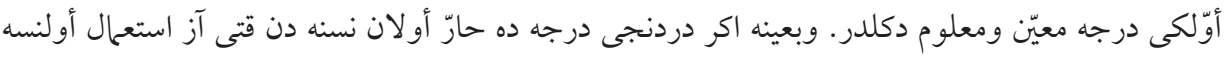

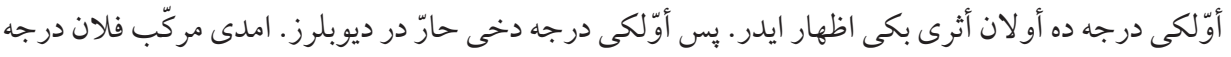

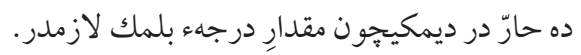
وصاحب موجز مطلقاً ديمشدر كه اول مفرددن

r

مستعمل أولان كميّت اول درجه نك كميّتى مقدارى در. أمّا اسحاق (الاسرائيلى)؛ ذكر اتمشدر كه بو مطلوبى

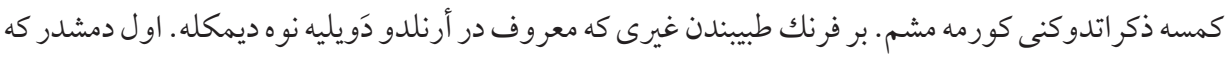

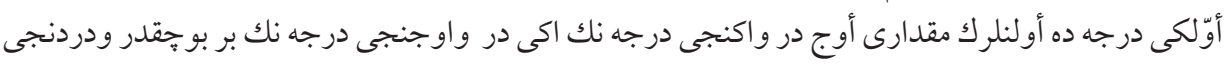

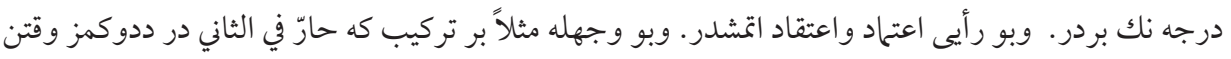

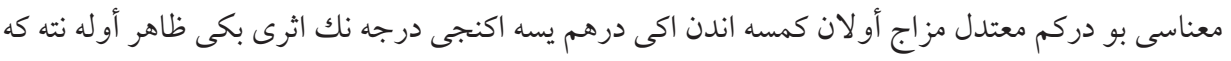

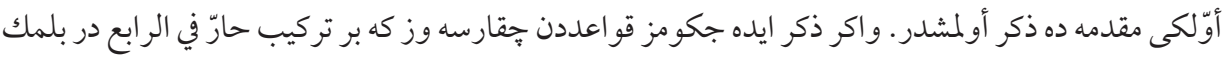

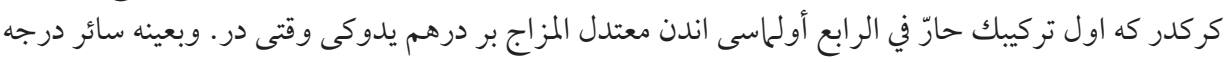




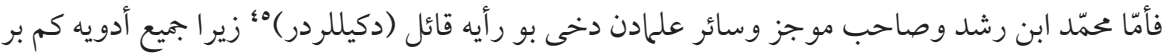

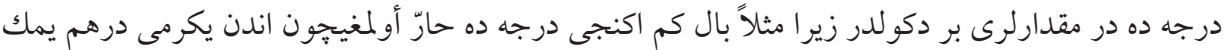

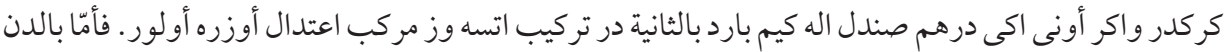

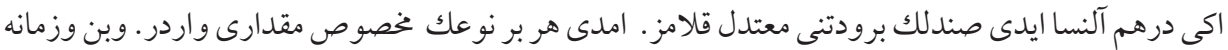

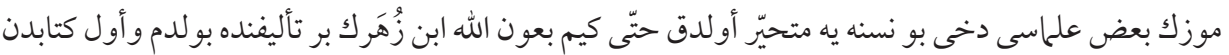

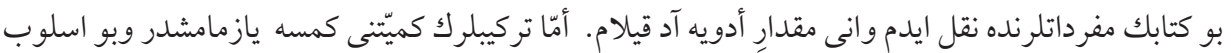

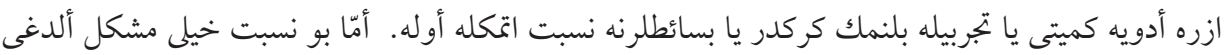

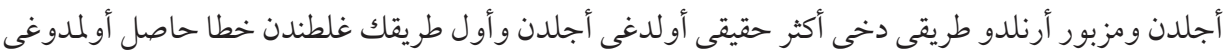

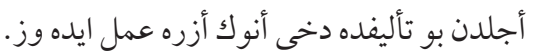
دردنجى مقدّمه أولدر كه هر بر درجه نك أجز الرنى

\section{r}

بلمك يعنى كيفيتلنى. وغورذو وعلمانك اكثرى ظنّ اتمشلركيم نسبت اجزاء كيفيه على نسبة الضعف المتو الية

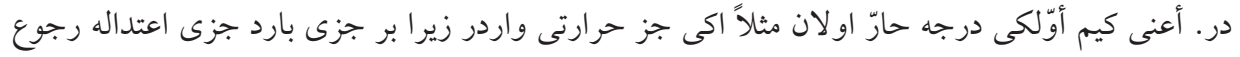

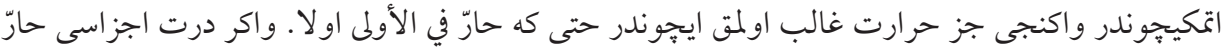

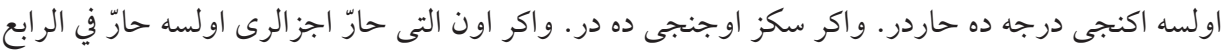

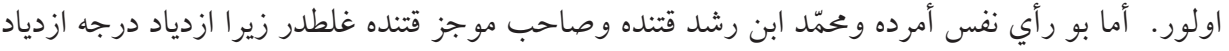

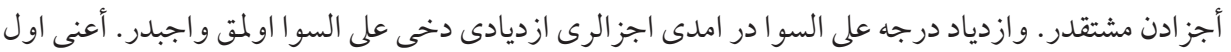

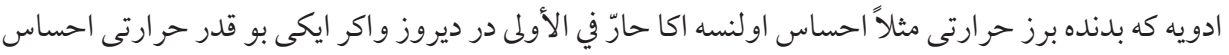

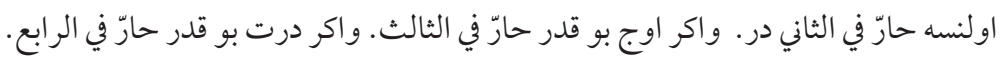

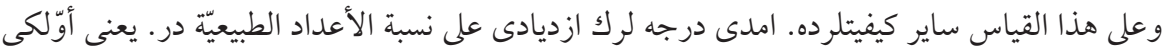

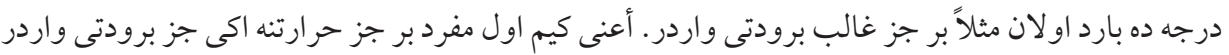

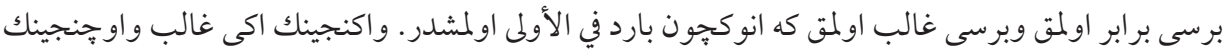

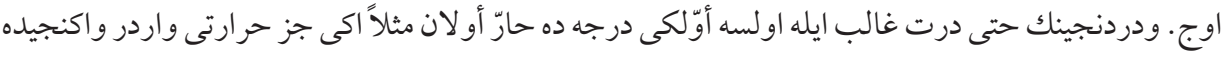

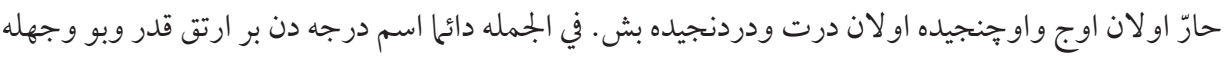

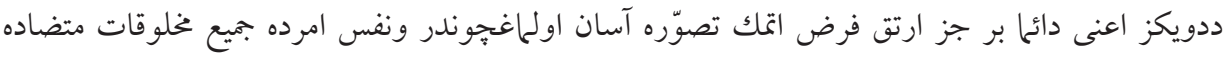

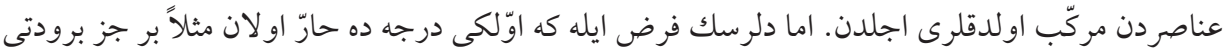

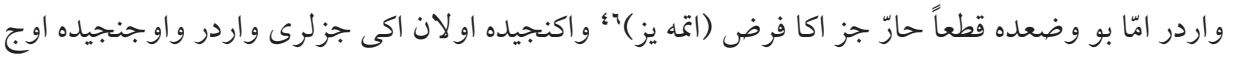
ودردنجيده درت امّا قطعا حر ارتلرى اولميه. واكى طريقك ورنك 


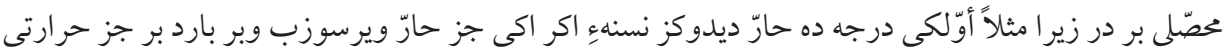

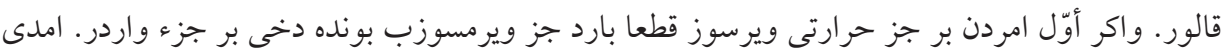
بونلرك يولى بردر.

فأمّا بز ايدرز أوّلكى علمانك رأيى كرجهه كه نفس أمرده فرض حقيقى اولميه اما حق نتيجيه بوجه آسان

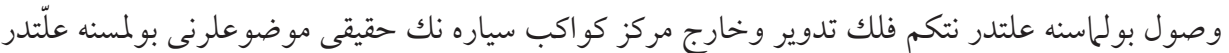

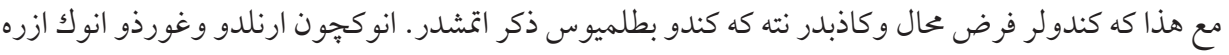

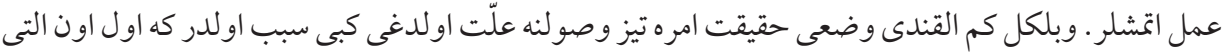

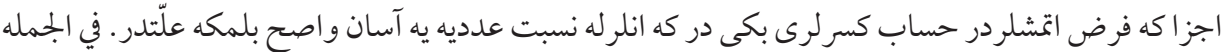

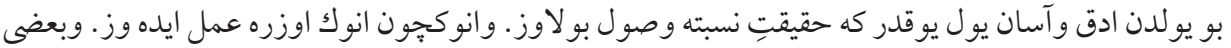

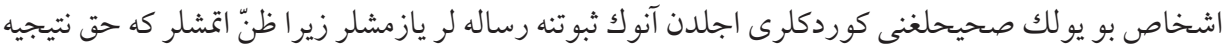

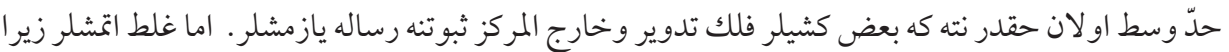

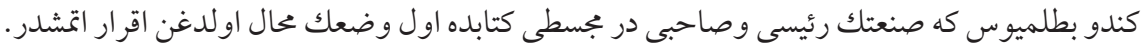
بشنجى مقدّمه بودر كه. ناقص اشتر اكى واجتماعى اكمله نقصان ايدر وبالعكس دخى اعنى ناقصه اكملدن

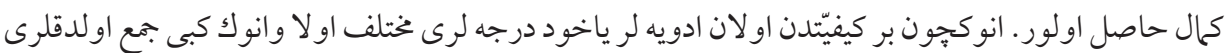

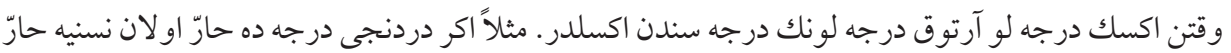

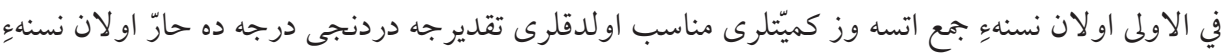

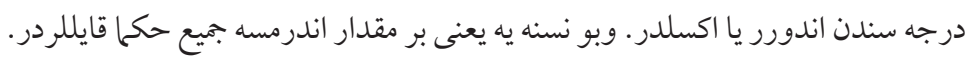

ومشاهده يله دخى ظاهردر زيرا قتى اسّى صويه اليجق صو قاتسوز البته اسّيلغى اكسلدر اما نه درجه ده يا

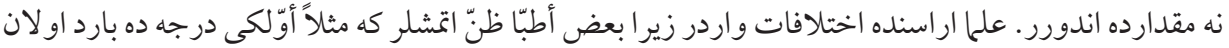

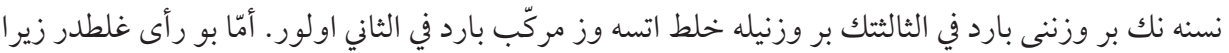

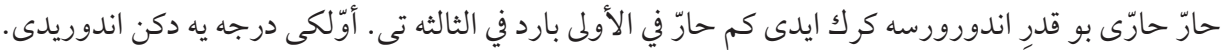

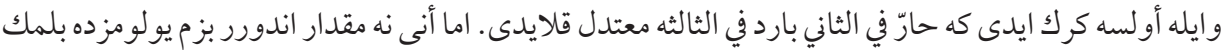

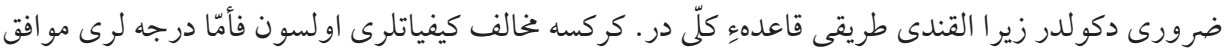

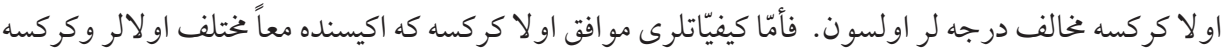

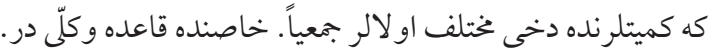
فامّا ناقص ادويه معتدل اولسه أعنى كه غيرى معتدل مفرداتلريله معتدل ادويه جمع اولنسه اكا دخى قاعده

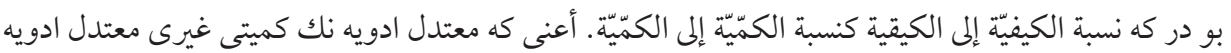

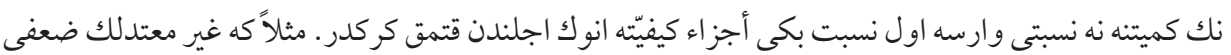




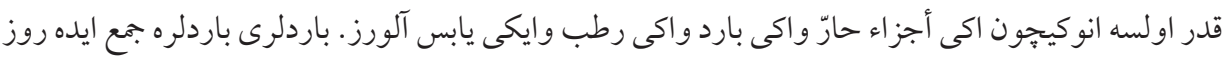

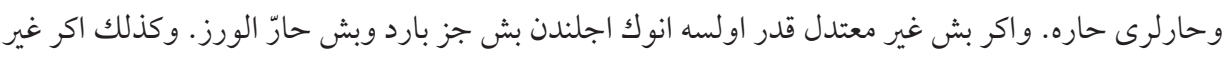

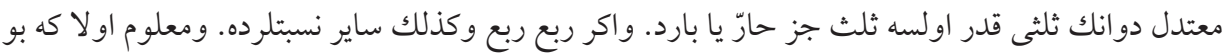

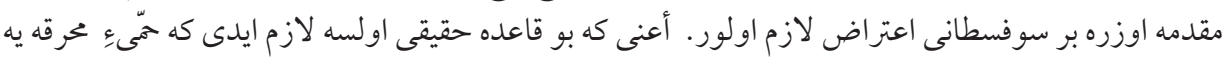

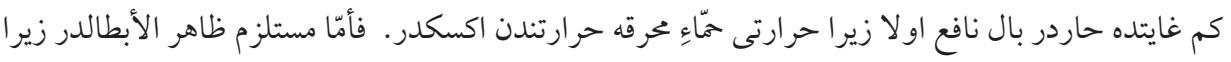

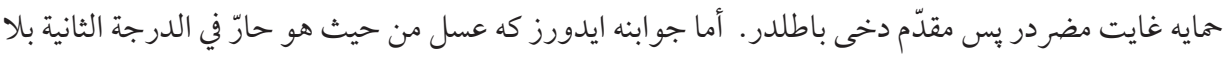
شك كيم حمايه من حيث مضرد بـ مايث

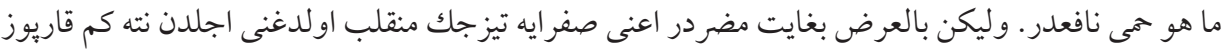

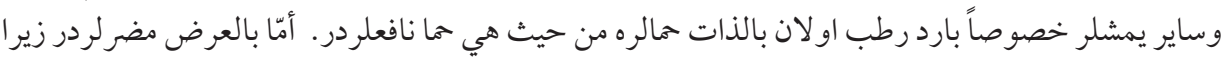
تيز جك متعفّن اولورلر.

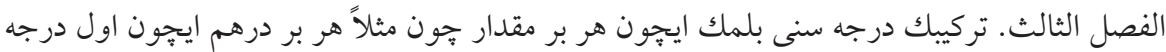

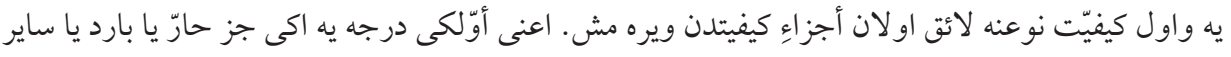

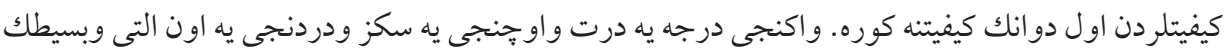

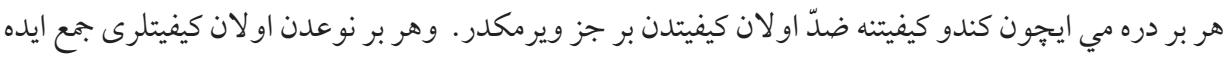

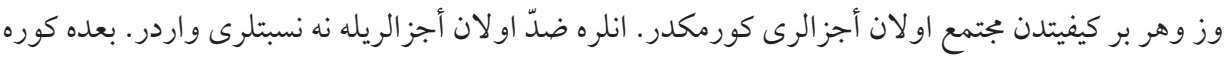

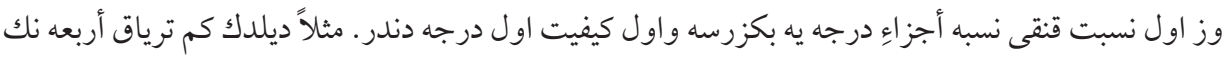

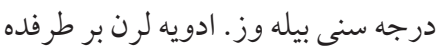




\begin{tabular}{|c|c|c|c|c|c|}
\hline درهم & أجزاء رطبه & أجزاء يابسه & أجزاء بارده & أجزاء حارّه & أسماء أدويه \\
\hline 0 & 0 & $\varepsilon \cdot$ & 0 & 7. & زراوند \\
\hline 0 & 0 & 7. & 0 & $\varepsilon \cdot$ & جنطيانا \\
\hline 0 & 0 & $\varepsilon \cdot$ & 0 & $\varepsilon \cdot$ & حبّ الغار \\
\hline 0 & 0 & 7. & 0 & 7. & 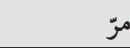 \\
\hline 7. & 7. & $r \varepsilon$. & 7. & $r \varepsilon$. & عسل \\
\hline $1 \cdots$ & $1 \cdots$ & $\varepsilon \varepsilon$. & $\wedge$. & $\varepsilon \varepsilon \cdot$ & جمله \\
\hline
\end{tabular}

يازدق ثم هر بر كيفيتلرك أجزالرى وميزان درهم. بعده جمع اتدك هر بر نوعدن اولان كيفيتلرى باشقه.

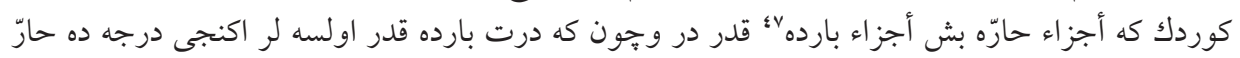

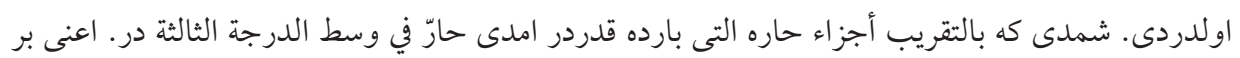

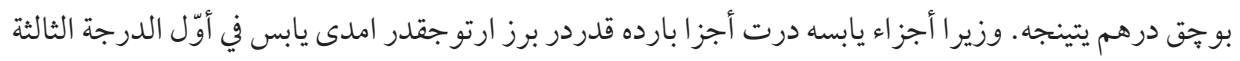
در. اعنى اكى درهمه يقين يتينجه.

\begin{tabular}{|c|c|c|c|c|c|c|}
\hline \multicolumn{7}{|c|}{ تركيب فلاسفه نك كيفيتى درجه سى } \\
\hline يأجزه & رأجزاء & أجزاء & حاره & وزنلرى & طبيعتلرى & أسماء أدويه \\
\hline 17. & 1. & 1. & 17. & • & ح ي في الرابع & فلفل \\
\hline$\varepsilon \cdot$ & 1. & 1. & $\wedge$. & 1. & حارّ في الثالثة يابس في الثانية & دار فلفل \\
\hline$\Lambda$. & 1. & 1. & $\wedge$. & 1. & 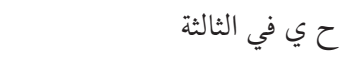 & قرنفل \\
\hline$r \cdot$ & 1. & 1 . & $\varepsilon \cdot$ & 1. & حارّ في الثانية يابس في الأولى & زنجبيل \\
\hline$r \cdot$ & 1. & $r \cdot$ & 1. & 1. & 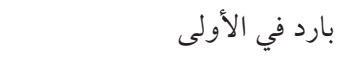 & املج \\
\hline$\varepsilon$. & 1. & $r \cdot$ & 1. & 1. & ب في الأولى ي في الثانية & بليلج \\
\hline
\end{tabular}




\begin{tabular}{|c|c|c|c|c|c|c|}
\hline 0 & $r \cdot$ & 0 & $r \cdot$ & 0 & حارّ يابس في الثانية & بابونج \\
\hline$\varepsilon \cdot$ & $1 \cdot$ & $1 \cdot$ & $\varepsilon \cdot$ & 1 . & ح ي في الثانية & أصل بابونج \\
\hline ᄉ. & $1 \cdot$ & 1. & $\Lambda$. & 1. & ح حي في الثالثة & دارجينى \\
\hline$\varepsilon \cdot$ & $1 \cdot$ & $1 \cdot$ & $\varepsilon \cdot$ & 1. & ح ي في الثانية & زرده هوب \\
\hline $1 \cdot$ & $r$. & 1. & $r \cdot$ & 1. & ح ر في الأولى & خصيه الثعلب \\
\hline Ir. & $r$. & $r$. & Ir. & $r$. & ح ي في الثانية & قزل أوزم منقى \\
\hline ᄉ. & 1. & 1. & $\varepsilon$. & 1. & ح في ب ي في ج & 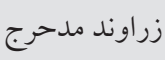 \\
\hline A. & 1. & 1. & $r \cdot$ & 1. & ح في الأولى & جوز هندى \\
\hline$\wedge \cdot$ & 1. & 1. & $\wedge$. & 1. & ح ي في الثانية & شيطرنج \\
\hline 7 . & $r$. & r. & 7. & $r$. & ح ي في الأولى & 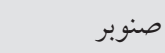 \\
\hline rtr. & 000 & 000 & Trt. & 000 & ح ي في الثانية & 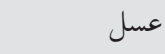 \\
\hline$r \mid r$. & vя. & $v_{7}$. & rar. & \multicolumn{2}{|c|}{ اشبو مركّب حارّ يابس في آخر الثانيةدر } & جمله \\
\hline
\end{tabular}

19

\begin{tabular}{|c|c|c|c|c|c|c|}
\hline أجزاء يابسه & أجزاء راطبه & أجزاء بارده & أجزاء حارّه & طبيعت & وزن درهم & أجزاء معجون فلونيا \\
\hline 1 . & 0 & 0 & $r \cdot$ & حrي ا & 0 & زعفران \\
\hline rr. & $r \cdot$ & $r \cdot$ & rr. & ح ي في ع & $r \cdot$ & فلفل \\
\hline$\wedge$. & $r \cdot$ & $r \cdot$ & $\wedge$. & ب ي في r & $r \cdot$ & بزر بنج \\
\hline 17 & 1 . & 1. & 17. & ب ي في ع & $1 \cdot$ & افيون \\
\hline$\wedge$ & 1 & 1 & $\wedge$ & ح ي في & 1 & فطر اساليون \\
\hline 17 & 1 & 1 & 17 & ح ي فيء & 1 & فزفيون \\
\hline$\varepsilon \cdot$ & 0 & 0 & $\varepsilon \cdot$ & ح حي في r & 0 & دهن بلسان \\
\hline$V \varepsilon \varepsilon$ & 117 & 117 & $V \varepsilon \varepsilon$ & & 117 & عسل \\
\hline Irre & $r \varepsilon \wedge$ & $r \varepsilon \wedge$ & IrAN & يب أوّل الثالثة & حارّ يابس & جمله \\
\hline
\end{tabular}




\begin{tabular}{|c|c|c|c|c|c|c|}
\hline يابسه أجزاء & أجزاء & أجزاء & حارّه & طبيعت & درهم & أجزاء أيارج فيقرا \\
\hline$\wedge$ & r & r & $\wedge$ & ح ي في r & r & مصطكى \\
\hline$\varepsilon$ & r & r & $\wedge$ & حrي 1 & r & زعفران \\
\hline$\wedge$ & r & r & $\wedge$ & ح'يا & r & سنبل \\
\hline$\wedge$ & r & r & $\wedge$ & ح ي في r & r & حبّ بلسان \\
\hline 17 & r & r & ir & ح ي في r & r & اسارون \\
\hline 17 & r & r & ir & ح ي في r & r & سليخه \\
\hline 17 & r & r & 17 & ح ي في r & r & دار جينى \\
\hline$\wedge$ & r & r & $\wedge$ & ح ي في & r & ادخر \\
\hline$I Y \Lambda$ & 17 & 17 & $1 Y \wedge$ & ح ي في r & ir & صبر \\
\hline 97 & $r \varepsilon$ & $r \varepsilon$ & 97 & ح ي في r & $r \varepsilon$ & عسل - عل \\
\hline$r \cdot \wedge$ & 07 & 07 & rir & في أوّل الدرجة الثالثة & حارّ ويد & جمله \\
\hline
\end{tabular}

\begin{tabular}{|c|c|c|c|c|c|c|}
\hline أجزاء يابسه & أجزاء راطبه & أجزاء بارده & أجزاء حارّه & طبيعت & درهم & أجزاء برش \\
\hline rr. & $r \cdot$ & $r \cdot$ & rr. & ح ي في ع & $r \cdot$ & فلفل أبيض \\
\hline$r r$. & $r \cdot$ & $r \cdot$ & rr. & ح ي في ع & $r \cdot$ & فلفل أسود \\
\hline$\wedge$. & $r \cdot$ & $\wedge$. & $r \cdot$ & ح حي في & $r \cdot$ & بزر بنج \\
\hline 17. & 1. & 17. & 1. & ح ي في ع & 1. & افيون \\
\hline $1 \varepsilon$ & v & $\checkmark$ & rA & ح في r ي في 1 & v & زعفران \\
\hline 7 & اونصف & اونصف & r & ح في اي في r & اونصف & سنبل \\
\hline$r \varepsilon$ & اونصف & اونصف & $r \varepsilon$ & ح حي في & اونصف & فرفيون \\
\hline ir & اونصف & اونصف & ir & ح ي في r & اونصف & جندبيدستر \\
\hline ir & اونصف & اونصف & Ir & ح حي في & اونصف & عاقر قرقرحا \\
\hline 997 & $r \leqslant q$ & $r \leqslant q$ & 997 & ح ي في r & $r \leqslant q$ & عسل \\
\hline $19 \varepsilon \varepsilon$ & rer & orr & 1200 & \multicolumn{2}{|c|}{ حارّ في الثانية يابس في الثالثة } & جمله \\
\hline
\end{tabular}




\begin{tabular}{|c|c|c|c|c|c|c|}
\hline أجزاء يابسه & أجزاء راطبه & أجزاء بارده & أجزاء حارّه & طبيعت & درهم & أجزاء سفوف \\
\hline 7. & VV Vونصف & Vونصف & $r \cdot$ & ح ي في r & Vاونصف & سقمونيا \\
\hline$v \cdot$ & عونصف & عونصف & $v \cdot$ & ح ي في ع & عونصف & فلفل أبيض \\
\hline rt & عونصف & عونصف & rt & ح ي في r & عونصف & قاقوله \\
\hline ir & اونصف & اونصف & Ir & ح ي في r & اونصف & زنجبيل \\
\hline Ir & اونصف & اونصف & Ir & ح ي في r & اونصف & دار هينى \\
\hline 7 & اونصف & اونصف & 7 & ح ي في & اونصف & بسباسه \\
\hline ir & اونصف & اونصف & ir & ح ي في r & اونصف & قرنفل \\
\hline 7 & اونصف & اونصف & 7 & ح ي في r & اونصف & جوز بوا \\
\hline r & اونصف & r & اونصف & ح في في & اونصف & املج \\
\hline 0. & $1 \cdots$ & 0. & $1 \cdots$ & ح ي في 1 & 0 & سكر أبيض \\
\hline$\varepsilon r$. & 1.0 & 1.0 & $\varepsilon r$. & ح & 1.0 & 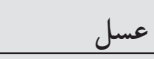 \\
\hline TAV & rr. & IAr & vro & لثالثة يابس في وسط الأولى & حارّ في آخر & جمله \\
\hline
\end{tabular}

IV

\begin{tabular}{|c|c|c|c|c|c|c|}
\hline أجزاء & أجزاء & أجزاء & أجزاء & طبيعت & درهم & أجزاء معجون نوش \\
\hline$r \varepsilon$ & 7 & Ir & 7 & ب ا ي r & 7 & ورد أحمر \\
\hline$\varepsilon \cdot$ & 0 & 0 & $\varepsilon$. & ح ي في r & 0 & سعد \\
\hline$r \varepsilon$ & r & $r$ & $r \varepsilon$ & ح ي في r & $r$ & قرنفل \\
\hline Ir & $r$ & r & ir & ح ي في r & $r$ & مصطكى \\
\hline Ir & $r$ & $r$ & 7 & ح ي ب 1 & $r$ & سنبل \\
\hline$r \varepsilon$ & $r$ & $r$ & $r \varepsilon$ & ح ي في r & $r$ & أسارون \\
\hline 17 & r & r & 17 & ح ي في r & r & قرفه \\
\hline 11 & r & r & 11 & ح ي في أوّل r & r & زرنب \\
\hline$\varepsilon$ & r & r & $\wedge$ & ح r 1 & r & زعفران \\
\hline$\wedge$ & r & r & $\wedge$ & ح ي في r & r & بسباسه \\
\hline 17 & r & r & 17 & ح ي r & r & قاقوله \\
\hline
\end{tabular}


Robert Morrison, Mūsā Calīnūs' Treatise on the Natures of Medicines and Their Use

\begin{tabular}{|c|c|c|c|c|c|c|}
\hline 17 & r & r & 17 & ح ي في r & r & هيل \\
\hline$\wedge$ & $r$ & $r$ & $\wedge$ & ح ي في r & $r$ & جوز بوا \\
\hline$r \cdots$ & $1 \cdots$ & $r \cdots$ & $1 \cdots$ & ب ي في & $1 \cdots$ & املج \\
\hline$r \cdots$ & $\varepsilon \cdots$ & $r \cdot$. & $\varepsilon \cdots$ & ح ي في 1 & $r \cdot$ & فانيذ \\
\hline 710 & orv & $\varepsilon \varepsilon r$ & 790 & وسط الأولى & حارّ ياب & جمله \\
\hline
\end{tabular}

\begin{tabular}{|c|c|c|c|c|c|c|}
\hline أجزاء يابسه & أجزاء راطبه & أجزاء بارده & أجزاء حارّه & طبيعتلرى درجه لرى & وزن درهم & مثروديطوس \\
\hline$\varepsilon \cdot$ & 1. & 1. & $\varepsilon \cdot$ & ح ي في r & 1 . & مر \\
\hline$r \cdot$ & 1. & $1 \cdot$ & $r \cdot$ & ح في & 1. & زعفران \\
\hline$\varepsilon \cdot$ & 1. & 1. & $\varepsilon \cdot$ & ح في في & 1. & غاريقون \\
\hline$\wedge$. & 1. & 1. & $\wedge$. & ح r & 1 . & زنجبيل \\
\hline$\wedge \cdot$ & 1. & 1. & $\wedge$. & ح ي في r & $1 \cdot$ & دارجينى \\
\hline$\wedge \cdot$ & 1. & 1. & $\wedge$. & ح في r & 1. & علك البطم \\
\hline$r \cdot$ & 1 . & 1. & $r \cdot$ & ح في 1 & 1. & كثيرا \\
\hline 17 & $\wedge$ & $\wedge$ & 17 & ج اي ا & $\wedge$ & سنبل هندى \\
\hline 17 & $\wedge$ & $\wedge$ & 17 & ح r & $\wedge$ & كندر ذكر \\
\hline IrA & $\wedge$ & $\wedge$ & IYN & ح ي في ع & $\wedge$ & خردل أبيض \\
\hline rr & $\wedge$ & $\wedge$ & rr & ح في في & $\wedge$ & عيدان البلسان \\
\hline rr & $\wedge$ & $\wedge$ & Ir & ح' اي r & $\wedge$ & اسطوخودوس \\
\hline rr & $\wedge$ & $\wedge$ & rr & ح ي في r & $\wedge$ & ادخر \\
\hline rr & $\wedge$ & $\wedge$ & $T \varepsilon$ & r & $\wedge$ & قسط \\
\hline$T \varepsilon$ & $\wedge$ & $\wedge$ & $T \varepsilon$ & ح في في & $\wedge$ & ساساليوس \\
\hline$T \varepsilon$ & $\wedge$ & $\wedge$ & $T \varepsilon$ & ح ي في r & $\wedge$ & كمافيطوس \\
\hline$T \varepsilon$ & $\wedge$ & $\wedge$ & rr & r r r & $\wedge$ & قنّه \\
\hline rr & $\wedge$ & $\wedge$ & $T \varepsilon$ & ح r & $\wedge$ & راتينج \\
\hline rr & $\wedge$ & $\wedge$ & $T \varepsilon$ & ح r & $\wedge$ & دار فلفل \\
\hline
\end{tabular}




\begin{tabular}{|c|c|c|c|c|c|c|}
\hline rr & $\wedge$ & 17 & $\wedge$ & ب ا ب r & $\wedge$ & سيو فسطيداره \\
\hline $7 \varepsilon$ & $\wedge$ & $\wedge$ & $7 \varepsilon$ & ح ي في r & $\wedge$ & جند بيدستر \\
\hline rr & $\wedge$ & $\wedge$ & r & ح ي في r & $\wedge$ & جاوشير \\
\hline rr & $\wedge$ & $\wedge$ & rr & ح ي في r & $\wedge$ & ساذج هندى \\
\hline rr & $\wedge$ & $\Lambda$ & rr & ح اي & $\wedge$ & ميعه سائله \\
\hline $7 \varepsilon$ & V & V & $7 \varepsilon$ & ح ي في r & V & سليخه \\
\hline 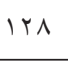 & V & V & $I Y \wedge$ & ح ي في ع & V & فلفل أبيض \\
\hline 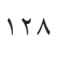 & V & V & IrA & ح ي في ع & V & فلفل أسود \\
\hline rr & V & V & rr & ح ي في r & V & سورنجان \\
\hline $7 \varepsilon$ & V & V & $7 \varepsilon$ & ح r r & V & جعده \\
\hline$I Y \Lambda$ & V & V & IrA & ح ي في ع & V & ثوم برى \\
\hline$r \varepsilon$ & V & V & $r \varepsilon$ & ح ي في r & V & دوقو \\
\hline 17 & V & V & 17 & ح ي في & V & اكليل الملك \\
\hline$r \wedge$ & V & V & 07 & r & V & جنطيانا \\
\hline$r \wedge$ & 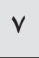 & V & 07 & r ح r & 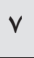 & دهن بلسان \\
\hline
\end{tabular}

وحب بلسان في بعض النسخة

IN

\begin{tabular}{|c|c|c|c|c|c|c|}
\hline أجزاء يابسه & أجزاء رطبه & أجزاء بارده & أجزاء حارّه & طبيعت & وزن & أجزا \\
\hline $11 \%$ & $v$ & v & $11 r$ & حي في ع & v & قوفيون \\
\hline rA & $v$ & v & rA & حي في r & v & مقل \\
\hline 17 & r & r & 17 & حي في r & r & سذاب \\
\hline$r \varepsilon$ & r & r & $r \varepsilon$ & حي في r & r & بزر سذاب \\
\hline 1. & 0 & ० & 1. & حي في & o & سنبل رومى \\
\hline$r \cdot$ & 0 & 0 & $r \cdot$ & ح ي في r & 0 & مصطكى \\
\hline 1. & 0 & 0 & 1. & ح ي في & o & صمخ عربى \\
\hline$\varepsilon \cdot$ & 0 & 0 & $\varepsilon \cdot$ & ح ي في r & 0 & فطر أساليون \\
\hline
\end{tabular}




\begin{tabular}{|c|c|c|c|c|c|c|}
\hline$\varepsilon$. & 0 & 0 & $\varepsilon$. & ح ي في r & 0 & قردمانا \\
\hline$\Lambda$. & 0 & $\wedge$. & 0 & ب ي في ع & 0 & أفيون \\
\hline$r \cdot$ & 0 & 0 & $r \cdot$ & ح ي في r & 0 & بزر رازيانج \\
\hline 1. & 0 & 0 & 1. & ح ي في & 0 & أحمر \\
\hline$\varepsilon$. & 0 & 0 & $\varepsilon \cdot$ & ح ي في r & 0 & كطر امشيج \\
\hline$r \varepsilon$ & $r$ & $r$ & Ir & r r r & $r$ & أنيسون \\
\hline 7 & $r$ & r & 7 & ح ي في r & r & \\
\hline$\varepsilon \wedge$ & $r$ & $r$ & $\varepsilon \wedge$ & ح في في & $r$ & فو \\
\hline$r \varepsilon$ & r & r & $r \varepsilon$ & ح ي في r & $r$ & مو \\
\hline Ir & r & r & $r \varepsilon$ & r & r & كبينج \\
\hline$r \varepsilon$ & r & r & $r \varepsilon$ & ح في r & r & أسارون \\
\hline ع ونصف & $1 \cdot$ & 1 . & ع و نصف & ب اي r & ع و نصف & أقاقيا \\
\hline 9 & ع ونصف & ع ونصف & 1. & I & ع ونصف & سرّه سقنقور \\
\hline 11 & ع ونصف & ع ونصف & 11 & ح ي في r & ع ونصف & هيوفاويقون \\
\hline $1 \cdots$ & ro & ro & $1 \cdots$ & ح ي في r & ro & شراب عتيق \\
\hline$r q \wedge \varepsilon$ & 997 & 997 & 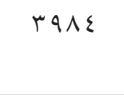 & ح ي في r & 997 & الجمل ثلاثة أمثال \\
\hline
\end{tabular}

امدى أجزاء حارّه وبارده ويابسه وراطبه بشقه بشقه جمع اولسه لر بلورز كه جمله أجزاءِ حاره بالتقريب درت

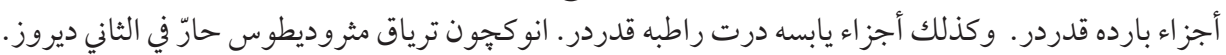

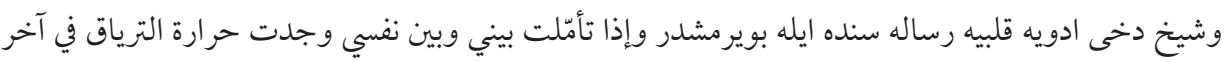

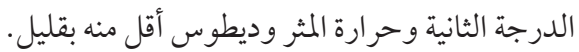

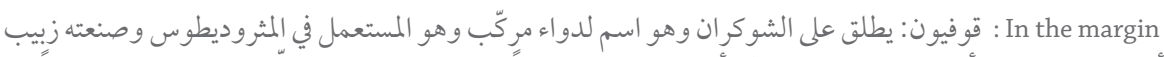

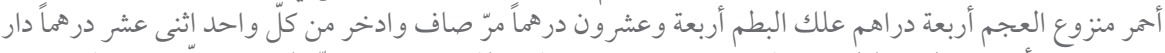

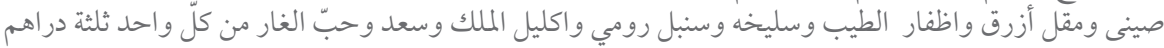

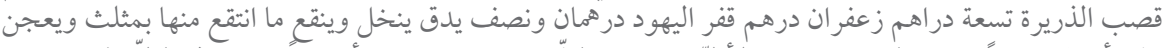

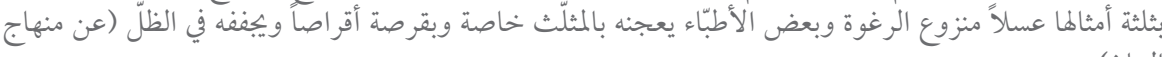
ودر بعضى نسخت قفر اليهود نيست ودر بعضى نسختها دو درمسنگ دونيم اسارون است ودر نستختى ديخر دو 
NAZARIYAT Journal for the History of Islamic Philosophy and Sciences

ب1

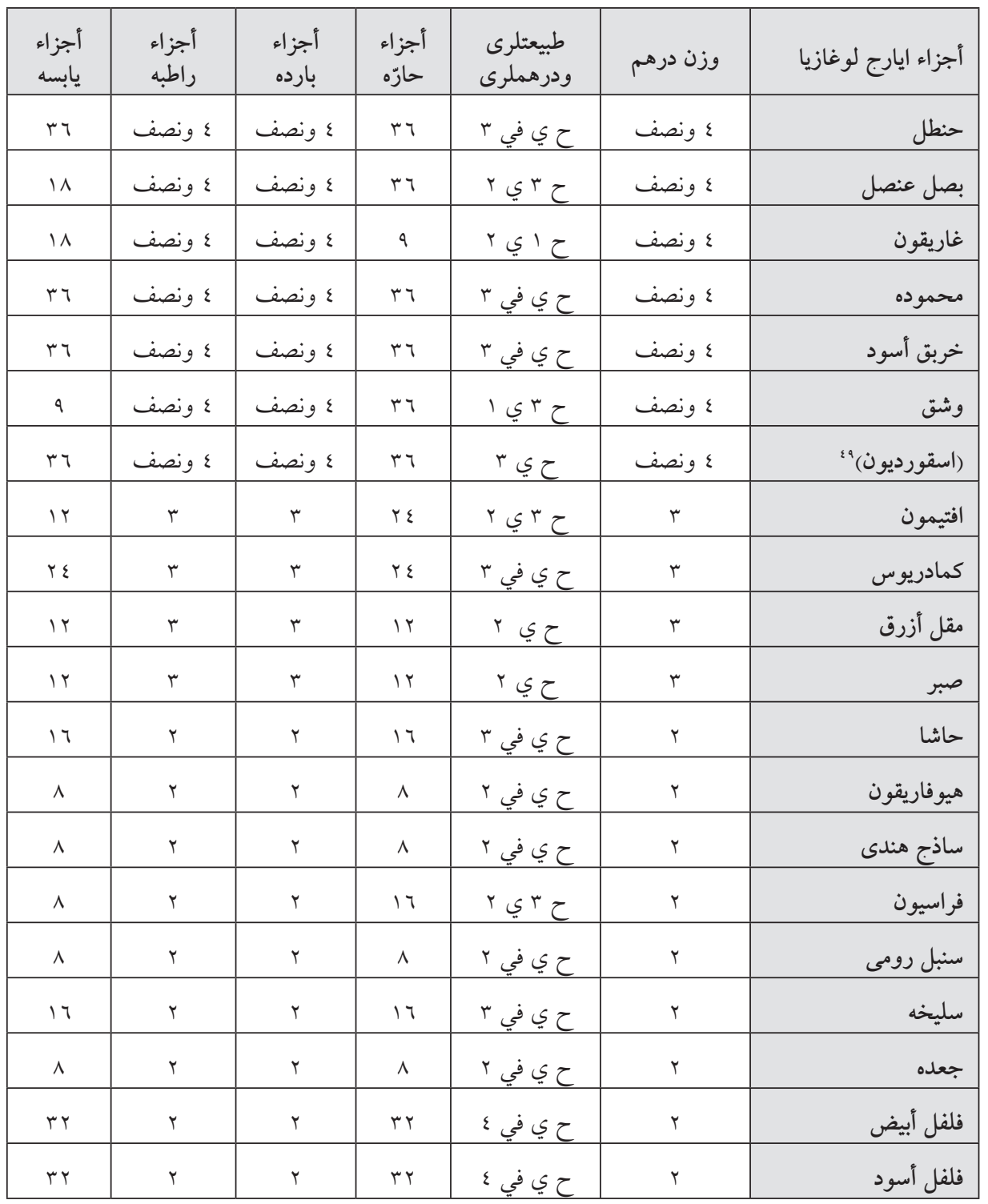

ي : اسقودريون

49

98 


\begin{tabular}{|c|c|c|c|c|c|c|}
\hline أجزاء يابسه & أجزاء راطبه & أجزاء بارده & أجزاء حارّه & طبيعتلرى & وزنلرى & لوغازيه أجزاء \\
\hline 17 & $r$ & r & 17 & ح ي في r & $r$ & دار فلفل \\
\hline$\varepsilon$ & $r$ & r & $\varepsilon$ & ح ي في & r & زعفران \\
\hline 17 & $r$ & r & 17 & ح ي في r & $r$ & دار هينى \\
\hline 17 & $r$ & r & 17 & ح في في & r & جاوشير \\
\hline$\wedge$ & $r$ & $r$ & 17 & r ح r & $r$ & بسفايج \\
\hline$\wedge$ & r & r & 17 & ح ح & r & سكبينج \\
\hline$\wedge$ & $r$ & r & 17 & r & r & جندبيدستر \\
\hline$\wedge$ & $r$ & $r$ & $\wedge$ & ح ي في r & $r$ & مرّ \\
\hline$\wedge$ & r & $r$ & $\wedge$ & ح في في & r & فطر اساليون \\
\hline$\wedge$ & $r$ & r & 17 & r & r & زراوند طويل \\
\hline$\wedge$ & $r$ & $r$ & $\varepsilon$ & ح اي r & r & افسنتين \\
\hline rr & r & r & ru & حي في ع & r & فرفيون \\
\hline$\wedge$ & r & $r$ & $\varepsilon$ & ح اي r & r & سنبل هندى \\
\hline$\wedge$ & r & $r$ & $\wedge$ & ح ي في r & $r$ & حماما \\
\hline$\wedge$ & r & $r$ & 17 & r ح r & r & زنجبيل \\
\hline Ir & 1 ونصف & 1 ونصف & Ir & ح ي في r & 1 ونصف & جنطيانا \\
\hline 7 & 1 ونصف & 1 ونصف & $r$ & ح اي r & ا ونصف & اسطوخودوس \\
\hline$\| r \wedge$ & rAY & rAr & $\| r \wedge$ & ح ي في r & rAr & عسل \\
\hline 1794 & rVr ونصف & rVT ونصف & $\mid V A$. & & $r V r$ & الحاصل \\
\hline
\end{tabular}

ايله اولسه بو دو اده حارّ أجز الر درت بجهق كرّه أجز اء بارده قدردر ودخى 
هף ارتوق ايله اولسه بو تركيب درجهء ثالثه نك أوّلنده حاردر. وكذلك أجزاء يابسه درت بجِق أجزاء راطبه

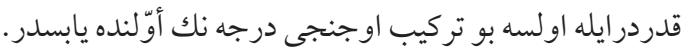

الفصل الرابع. بعده ايدورز اكر بلمك دلرسك كه هر بر درجه نك مخالفندن ساير درجه لو ادويه لردن نه

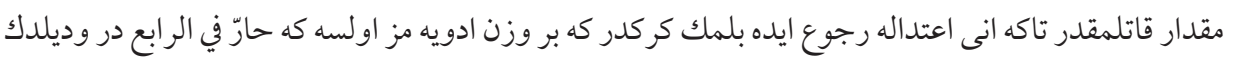

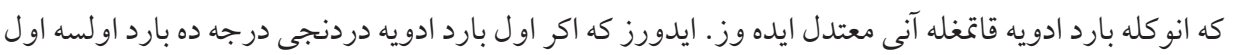

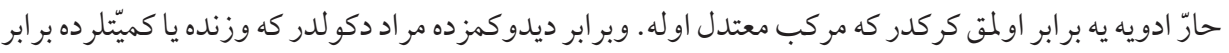

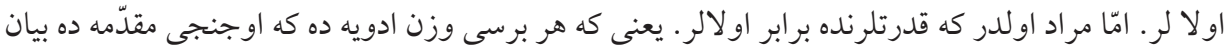

$$
\text { اولمشدر برابر اولالر. }
$$

و اكر بارد ادويه اوجنجى درجه ده بارد اولسه بر وزن ادويه وثلاثة أرباع وزن ادويه المق كركدر تا كيم اول

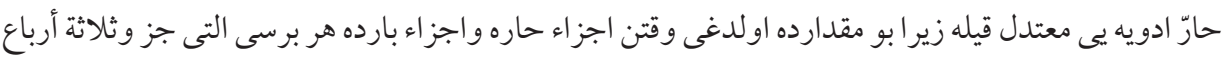

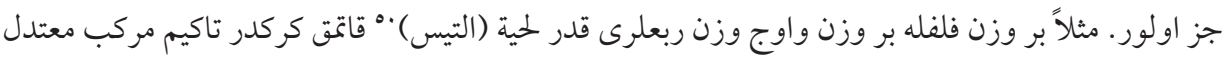

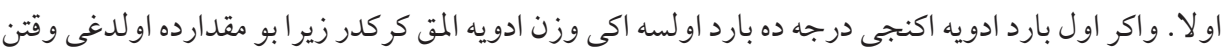

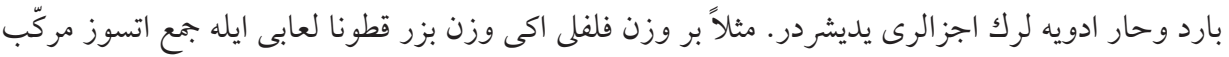

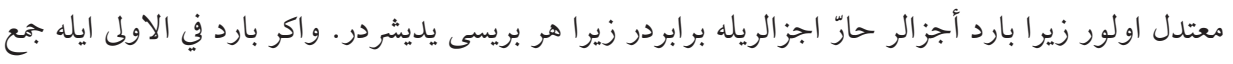

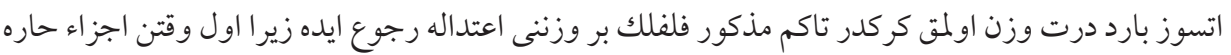

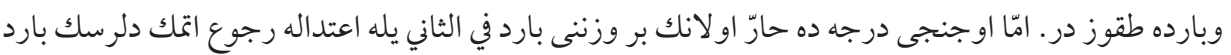

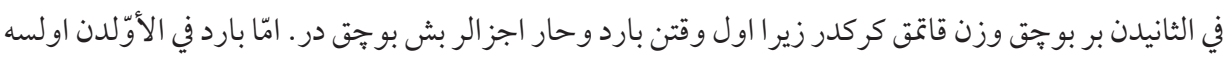

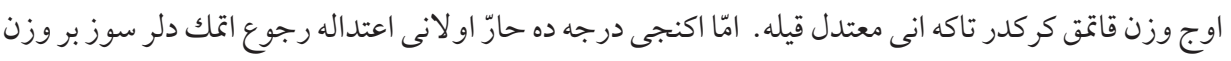

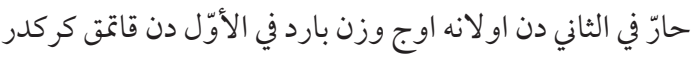

تاكه آنى معتدل قله زير ا ايله اولدغى وقتن اجزاء حارّ وبارده (يديشر ) '. و وعلى هذا القياس ساير كيفياتلرده. الفصل الخامس مباحثلرى بلدرر. آ اطبّا بيورمشلر كه اورام حاره ازديادلرى وقتنده رادعات محلّلات ايله

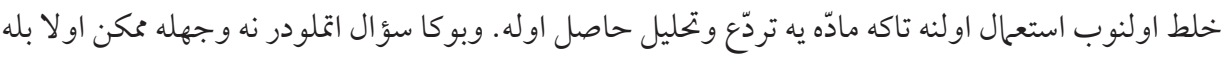

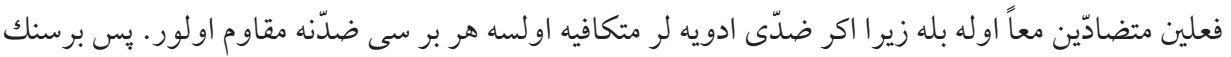


دخى تأثيرى ظاهر اولماز واويليه مركّب معتدل اولور ادى. و اكر برسى اقوى اولسه اقوى نك فعلى فعلى ظاهر اولور

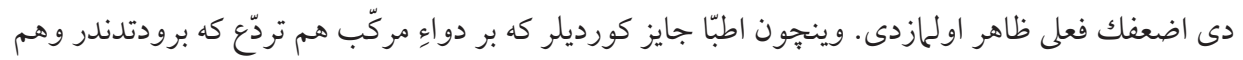

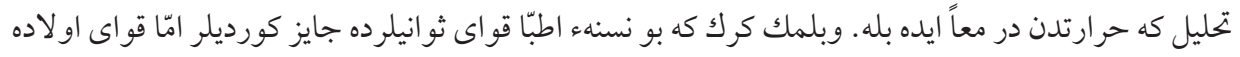

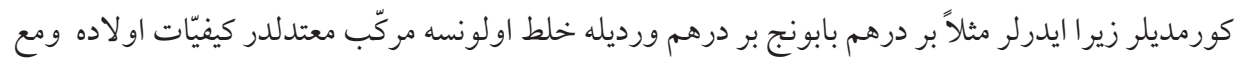

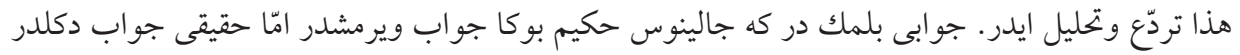

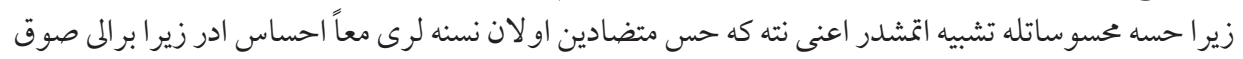

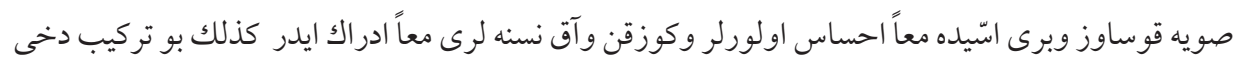

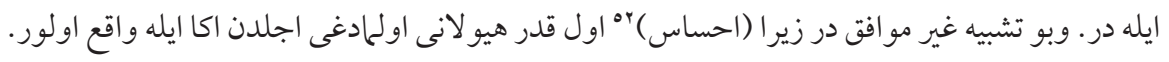

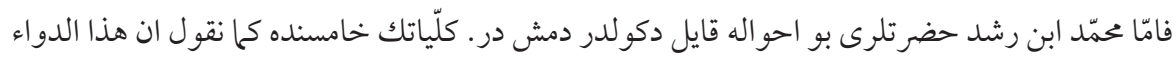

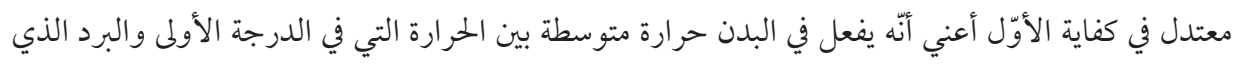

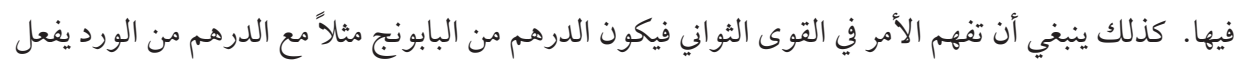

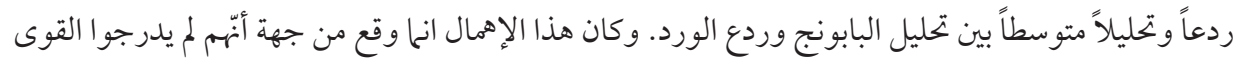

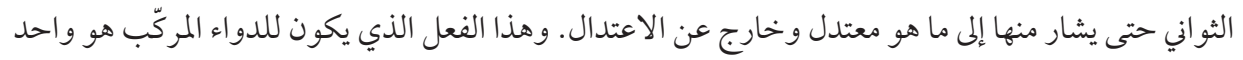

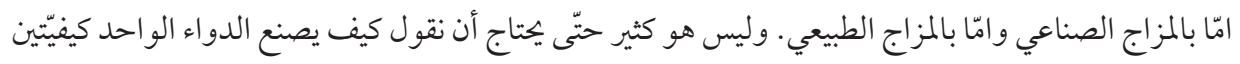

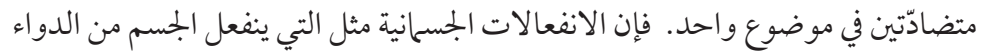

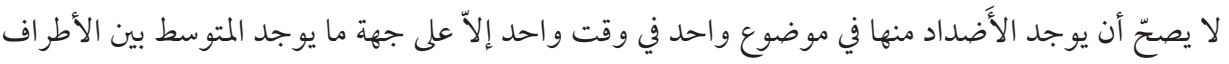

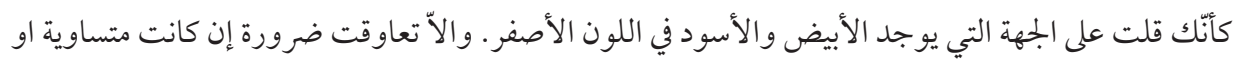

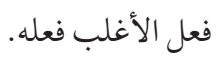

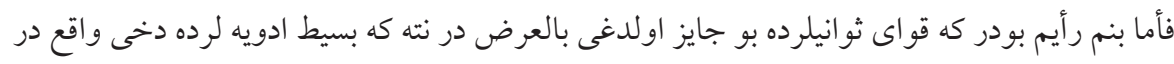

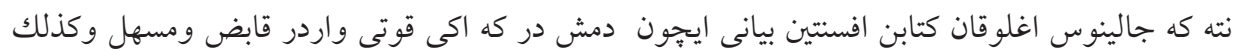

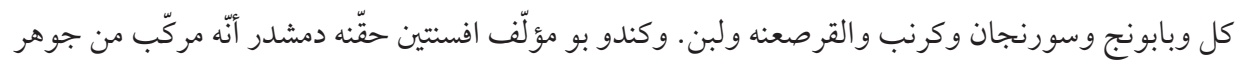

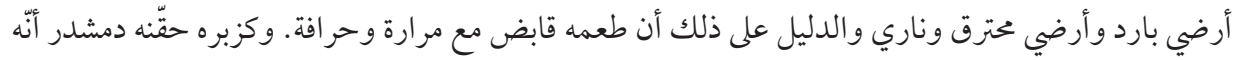

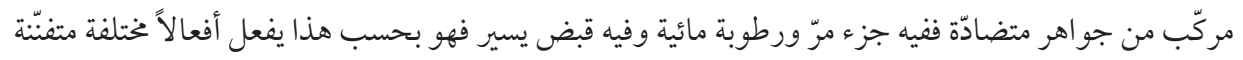

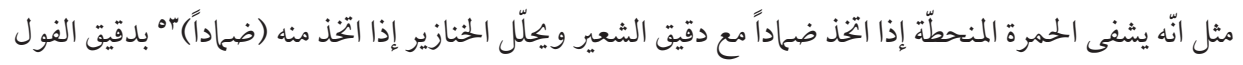

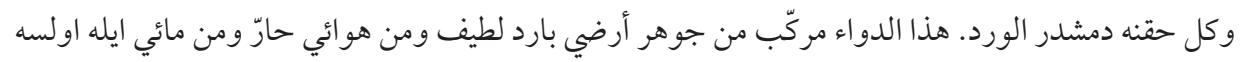


ايدورز جو نكم مفرد دو اده بو جائز اولدقدن صكره كه مفرد در. (وطبائعنك) ؛ه امتز اجيله متحد اولمشدر جونكم

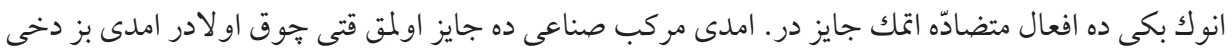

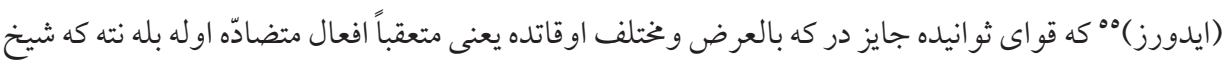

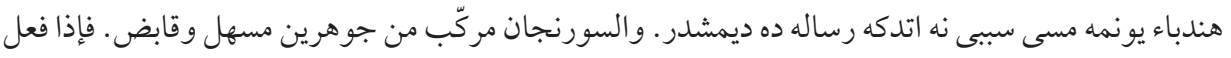

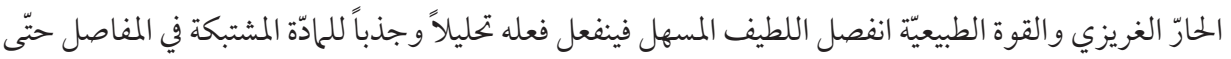

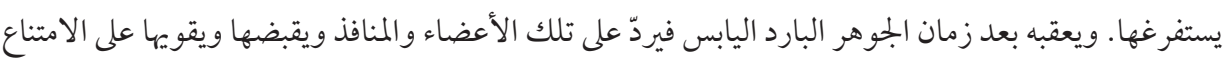

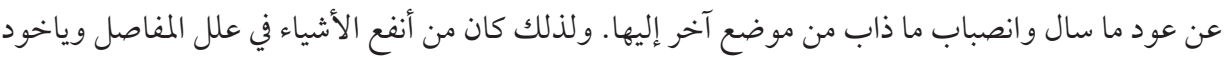
كه فاعل يعنى تركيب كه اتحاد غير حقيقيلى متحدّ اولمشدر وكذلك منفعل أعنى بدن يا عضو واحس ماحد حقيقى اولميه. فعل وانفعال دخى و احد بالحقيقة وحين و احدده اولمق لازم دكولدر.

انو كجون دخى شيخ مزبور رساله ده دمشدر وللبابونج قوة رادعة وقوة محلّلة. فإذا استعمل في الأورام

أو سقي في (الحميّات البلغمية الباردة المادّة)جّه فرقت الطبيعة بإذن خالقها تعالى القوتين. فاستعنت بالباردة على تطفية الحرارة الغالبة على الأعضاء فبالحر ارة على تحليل المادّة الباردة الجليدة هذا في الحمّىى. وامّا في الأورام فإنهّا توجّه القوة الباردة إلى المسالك والمنافذ فيضيّقها ويمنع المو ادّ منها وإلى المادّة المتوجّهة إلى العضو . ولما يحصل فيه فيه بعد فتردعها فتحذرها (فترحضها) vه. وتجمدها وتمنعها من السيلان الذي كان بها وإلى جوهر العضو فتلذّذها و تقويه فلا ينفعل عن الملدّة الخبيثة. وأمّا القوة الحارّة فتو جهرا إلى المادّة المتقربة في العضو حتّى تحلّ تلك المادّة

اكنجى سؤال بودر كه اوجنجى مقدّمه صادقه اولمزدى بياننده در زيرا ظاهرا كوررز كه بر مرتبه لو ادويه

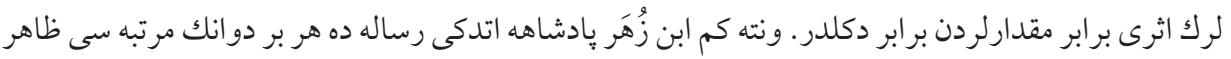
اولاجق مقدارى ذكر اتمشدر.

او جنجى سؤ ال بودر كه دردنجى مقدّمه حقيقى اولمزدى نته كه محمّد بن رشد كلّيّاتك بشنجى كتابنده بيان

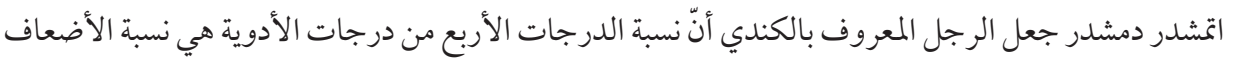
حتّى تكون الدرجة الرابعة ستة عشر ضعفاً. وذلك أنّه جعل الأوّل ضعف المعتدل والثانية ضعف الأولى و الثاالثة ضعف الثانية والرابعة ضعف الثالثة. فهالا كفاه في ذلك أن يقول إنّ الثانية ضعف الأولى والثالثة ودلثة ثلاثة أضعافها

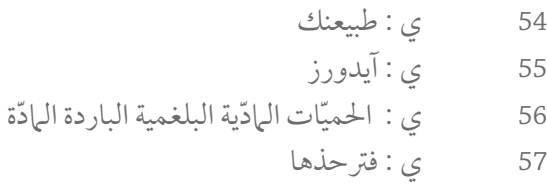


و الرابعة أربعة أضعافها. فإنّ هذا هو الذي قصد في ترتبها لتكون مراتبها متساوية. وذلك أنّهم أخذوا أوّل دواء

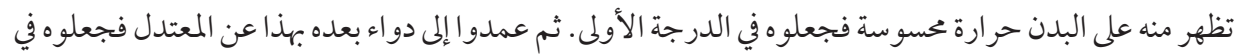
الثانية. فهذا لا شكّ هو ضعف الأوّل. ثمّ عمدوا إلى دواء بعده عن الثانية بعد الثانية عن الأوّل فجعلوه في الثالثة.

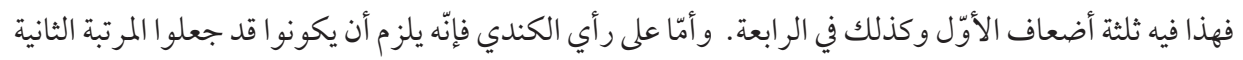

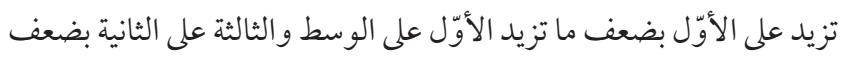

ما تزيد الثانية على الأوّل. فأيّ ضرورة ليت شعري كانت تدعو الأطبّاء إلى أن يتحفّظوا بهذه النسبة. وعلى هذا

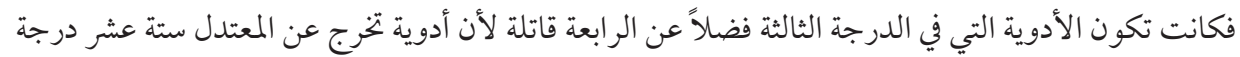
كيف حال الأبدان معها. و أيضاً فكان يكون بعد الدرجة الرابعة من الثالثة ليس بعد الثالثة من الثانية بل بعد الثالثة

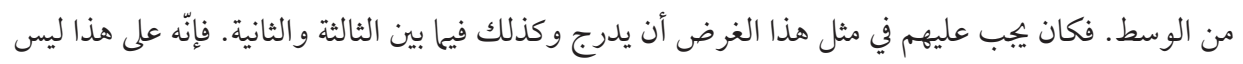
تكون مر اتب الدرج متساوية و أيّ الاختلال في هذا الصناعة أعظم من هذه الاختلال . وذلك أن ما قصد له من أوّل الأمر من (حفظ)^ه مر اتب زيادة القوى بعضها على بعض كان يفوتنا. وذلك أن المرتبة مثلاً التي نسبتها إلى الأوّل

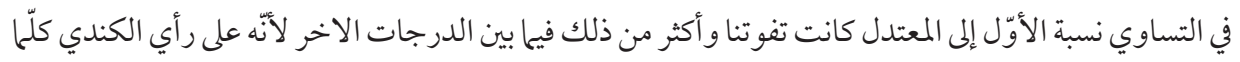
ارتفعت عظم العرض بينها حتّى لو كانت هنا درجة خامسة لكانت اثنين وثلاثين جزاً لأنّا كانت تزيد على لانه الر ابعة

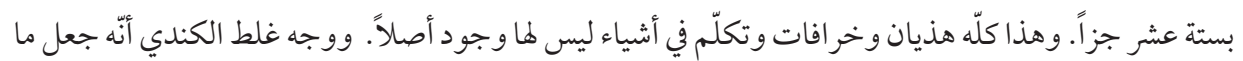

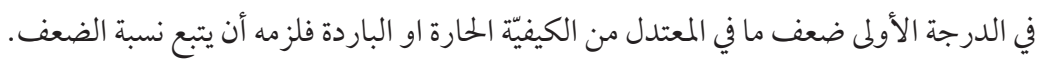
ولقائل أن يقول أن الذي قصدوا الأطبّاء بالدرجة الأولى هو ما يزيد على المعتدل جزء من ستة عشر . وعلى هذا إذا تركبت نسبة الضعف في زيادة الدرجات ليس يلزم أن يكون الدواء الذي في الدرجة الرابعة ستة عشر ضعناً

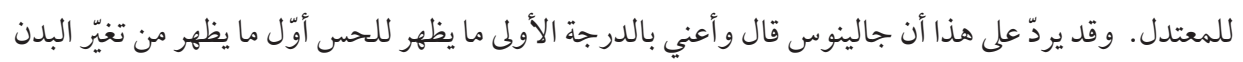
ولو كان المر اد بالدرجة الأولى ضعف المعتدل لم يكن التغيّر الذي يظهر في البدن منه أوّل تغير . فتأمّل هذا فهو بيّن. ولكن عادة الناس إذا غلط رجل مشهور أن يتبعوه لما غلب على طباعهم من قوة التقليد. دردنجى سؤ ال بودر كه بشنجى مقدّميه قايل اولونميه أعنى قانون كه 
أوّل مقدّمه ده ديدك كه أقل درجه لو ادويه اكثر درجه نك درجه سنى اندوره. مثلاً أقل حرارتى اولان نسنه أزيد

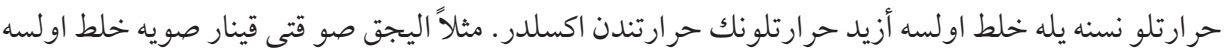

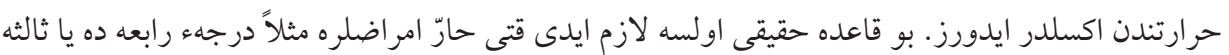

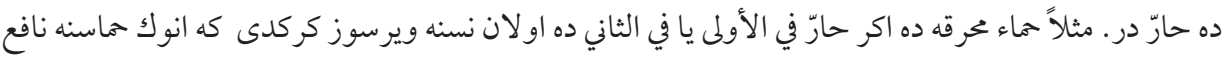
اولب اكسك ايده يدى. وبز كورورز كه اكا بغايت مضر در وينجه مضر أولماسوز . بز كورورز بعض بارد رطب دئ

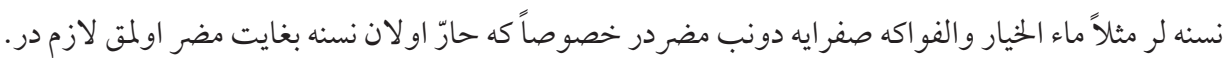
فأمّا بو نك جوابى ايجٌون ايدورز كه اختلاط اولندغى نسنه يله اكسى يا بالفعل اولا يا اكسى بالقوة او لا بو نسنه

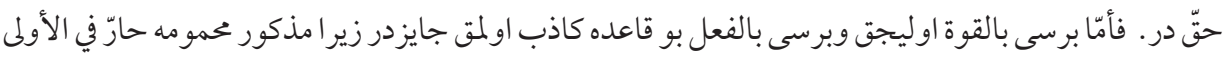
ويريجك يا بارد في الأولى دخى حالنجه اول دوا بدنده باقي اولسه بلكى نافع اولا يدى. فأمّا حرارت غرئ فريزيه

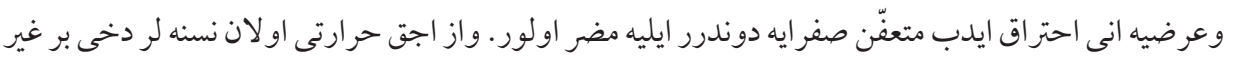
جهتدن دخى مضر در بالعرض زيرا حرارتى زياده ايدرلر بالعرض نته كه دمورجيلر اود اوزرنه برز صو سيرلر اود اقوى اولسون ديو زيرا هر ضد ضدنه مقاوم اولوب تقويته كلور. انو كجون حماء محرقه لرده ووبائلرده صوق

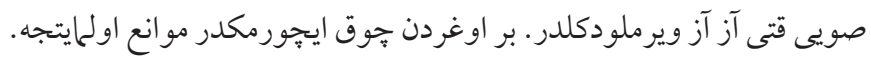
بشنجى سؤ ال دخى بشنجى مقدّمه ازره در. ايدورز كه جايز اولملاز كه ادنا درجه لو أعلا درجه لودن بو قدر

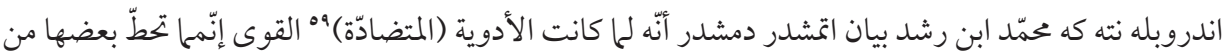
بعض بقدر ما فيها من تعادل التضادّ. أعني مثلاً أن الدواء البارد في الأولى إنّما يحطّ من الثاني في الحرارة بقدر ما

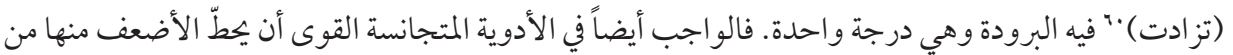
الأقوى

\section{I}

بقدر ما نسبة الضدّ في الدواء الأضعف إلى ضده أعظم نسبة منه في الدواء الأقوى. مثال ذلك أنّ الحارّ في الدرجة الأولى البارد فيه أعظم نسبة إلى الحارّ منه في الدواء الحارّ في الدرجة الثانية وفي الثالثة أصغر منه من الثانية. وإذا كان ذلك كذلك فالدواء المعتدل في الأدوية المتجانسة القوى هو أقرب المراتب في أن يحطّ ما فوقه إذا كانت نسبة المتضادّ فيه تقرب من أن يكون نسبة تعادل. ثمّ بعده ما كان في الدرجة الأولى ثم في الثانية ثمّ في الثالثة. 


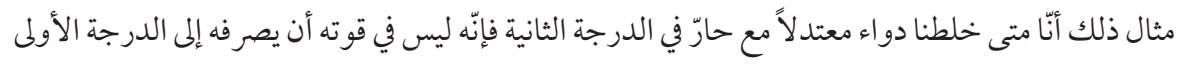

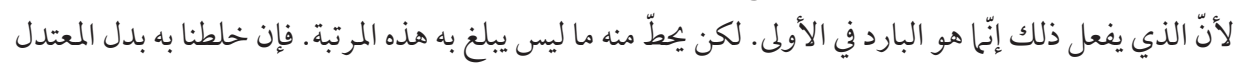

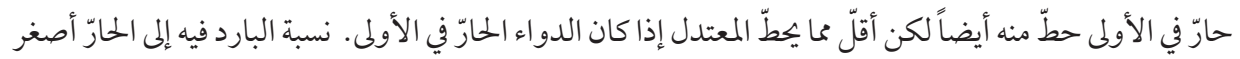

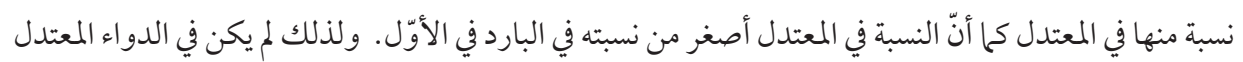

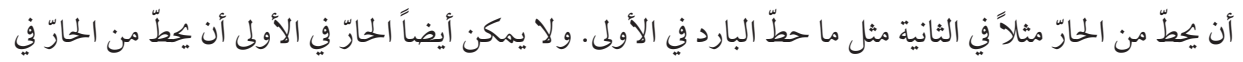

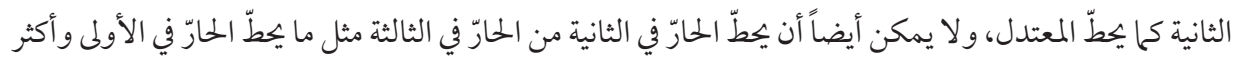

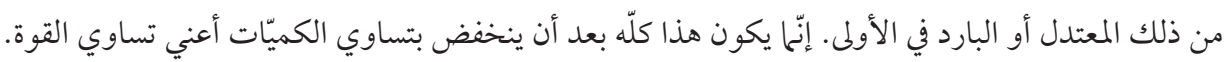

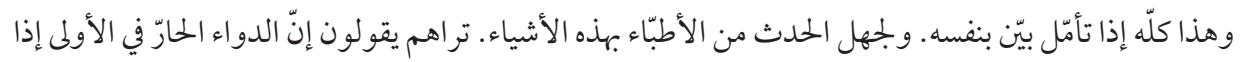

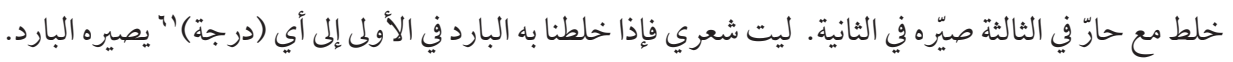

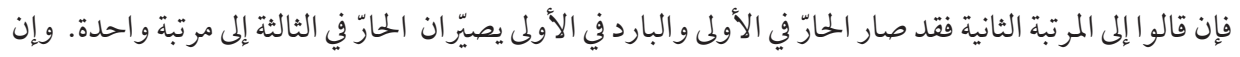

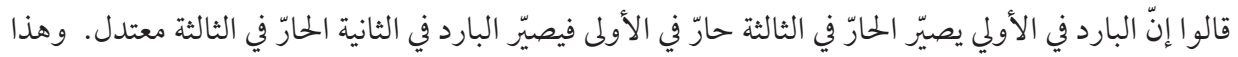

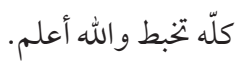


In the name of God, the merciful, the compassionate. Praise be upon all of the prophets.

Now then, we say that since man is assembled from the spiritual and the corporeal, knowledge of the science of medicine is thus, in one way or another, from among the divine religious observances. Therefore, they say, "The knowledge of bodies, then the knowledge of religions," because the antidotal cures are used according to the degree of the disease's quality to make the disease go away. Because sometimes we cure with compound [medicine]s, it is therefore necessary to know the compound's degree. Also, in this subject there are numerous perplexities and obstacles. On account of the best of men being the one who is of use to men, especially the chief physician and most virtuous scholar whom God singled out with the saintly soul and affable leadership and whom God granted success in achieving human perfections and the arrival of eternal happiness, I mean his excellency Ahi Çelebi, may God the exalted support and preserve him, who commanded that this humble servant, the least of the physicians, I, Mūsā Cālīnūs al-Isrā'ìlì, assemble the useful, summary, and exegetical epistle from the words of Islamic, Frankish, Greek, and Jewish physicians.

As for the degrees of the diseases' qualities and the measure of every single medicine, that is to say by using what measure might the degree's effect be apparent, and for each compound used, what measure of time remains so that its property might not be lost? It is necessary that I have mentioned in my book the degrees of the compounds that are used, the compassionate God willing.

The first chapter makes known, through intellectual division, ${ }^{62}$ the reasons for our making a compound, because we desire to communicate the compound's degree. The physicians' rule is this: Any time simple medicines are sufficient, we should not compound. Thus, to communicate the reasons for our making compounds and in order to facilitate their memorization, I have placed their divisions in the form of the tree. ${ }^{63}$

62 This could be a truncation of al-taksim va-'l-sabr (division and investigation).

63 On these tree diagrams, see Emilie Savage-Smith: "Medicine in Medieval Islam," in Michael H. Shank and David C. Lindberg (eds.), The Cambridge History of Science, (Cambridge and New York: Cambridge University Press, 2013) vol. 2: 139-67, at 148-9. 
$2 a^{64}$

Simple medicines

A. It [a simple medicine] might be its connection to treatment

A1. Or it might not be entirely beneficial for the purpose

A1a. It might not be entirely beneficial in essence

$\mathrm{Ala}_{1}$. It might be on account of it being very powerful

$A 1 a_{1 a}$. Or it might be on account of its properties being many and not what is intended

$A 1 a_{1 b}$. However, it might be on account of there being in the intended thing many properties that the compound is needed to weaken; only for our purpose is it not useful in essence

$\mathrm{Ala}_{2}$. Or due to its weakness

$\mathrm{Ala}_{2 \mathrm{a}}$. It might be weak from how many of its effects are entirely beneficial

$\mathrm{Ala}_{2 \mathrm{a} 1}$. These effects might be similar, just as Theriac's effect was compounded to benefit all of its offspring

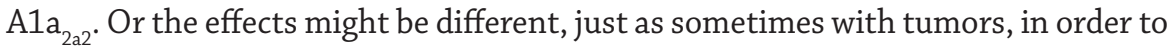
restrain, dissolve, and strengthen the organ, we compound some of the causes

$\mathrm{Ala}_{2 \mathrm{~b}}$. It may be that its only effect is also weak

$\mathrm{Ala}_{2 \mathrm{~b} 1}$. It might be weak in itself and in need of something to assist, like our mixing in salt, gum, agaric, ginger, and turpeth

$\mathrm{Ala}_{2 \mathrm{~b} 2}$. Or he should say that its being added to is on account of its being very ephemeral, so to make it slow down it should be mixed with something else, just as we mix oil with Balm of Gilead and wax

64 On this page, Ömer Şifai [attribution from Ekmeleddin İhsanoğlu (ed.), Osmanlı Tibbi Bilimler Literatürü Tarihi, (Istanbul: Ircica, 2008), vol. 1: 135] or Şa'bān Şifai [attribution from İzgi, Osmanlı Medreselerinde İlim, vol. 2, 23] wrote a marginal note. It begins: “These expository ornaments, that are in the picture of a tree, are far from the 100,000 ranks of the Canon because the purpose of the science of medicine is the preservation of health and the elimination of disease. A scholar and physician from whatever nation he may be, is obliged to be sympathetic and compassionate while being a scholar. Friendly and inimical treatments should be separated..." For more on Ömer Şifai, see B. Harun Küçük: "New Medicine and the Hikmet-i Tabī'iyye Problematic in Eighteenth-Century Istanbul," in Y. Tzvi Langermann and Robert G. Morrison (eds.), Texts in Transit (University Park, PA: The Pennsylvania State University Press, 2016): 222-42, at 227 and 233. If this marginal note is from the $966 \mathrm{AH}$ copyist, the author of the note is neither Ömer Şifai nor Şa'bān Şifai. 
$\mathrm{Ala}_{2 \mathrm{~b} 3}$. It should, however, be weak from arriving at the intended place, just as in viper ${ }^{65}$ theriac we mix the other medicines to send to the primary organs

A1b. Or accidentally

$\mathrm{A}_{1} \mathrm{~b}_{1}$. That is necessary, I mean that on account of how it is harmful to the friendly [medicine], according to me, compounding is needed in order to ameliorate

$\mathrm{Alb}_{2}$. Or that it is on account of it being beneficial to the inimical [medicine]

$\mathrm{Alb}_{3}$. However much it is harmful to the friendly or beneficial to the inimical, its benefit is with the effort for friendly [cures]. That is, a compound is needed to stave off the bitter and the ill-favored.

A2. It entirely serves the purpose and there is no need to compound [medicines] for the likes of this

A3. Or it might be doubtful whether or not it suffices completely, and it is permissible to compound similar parts for the likes of this

B. Or it may not be. And if there is no connection [to treatment] or suitability, then it is decidedly not the intention

$2 \mathrm{~b}$

The second chapter mentions some preliminaries necessary for knowing the rank of ${ }^{66}$ a compound. The first preliminary, which clarifies what the concept of a degree is, is this. It should be known that when a little of one thing's effect becomes apparent in the body, it is from a primary's qualities, I mean from heat or from cold or from moisture or from dryness. But this effect would not become apparent any faster even as far as it [the primary] was used repeatedly. However, the copiousness of its use means that the degree of this effect is the first. If the above-mentioned effect is stronger so that its effect might be apparent from the beginning, although this effect's temperament (mizāc) should not remain very subject to change and its effects' harm is not felt whatever an increase is being used, then this effect is of the second degree. And if the above-mentioned effect is stronger, so that its effects' ap-

65 This is a theriac prepared from a viper.

66 My translation of martaba as 'rank' follows Y. Tzvi Langermann: "Another Andalusian Revolt? Ibn Rushd's Critique of al-Kindi’s Pharmacological Computus,” in Jan P. Hogendijk and A.I. Sabra (eds.), The Enterprise of Science in Islam: New Perspectives, (Cambridge and London: MIT Press, 2003): 351-372, at 357. 
parent harms might be evident but not of a measure that shall cause death or corruption, then the quality of the effect of something like this is of the third degree. If the above-mentioned effect is of a measure that shall cause death or corruption, then this one is of the fourth degree. ${ }^{67}$

The second preliminary is this: As the quantities increase and are doubled, their qualities also increase accordingly. For example, sandalwood, of which two drams are cold in the second [degree]; if there are four drams, then there are four cold parts. One dram of pepper that is hot in the fourth has four hot parts. If they [the simples] are able to make an equilibrium, then a compound of them is equilibrate.

The third preliminary is this: Whatever measure of each medicine being used, its effect at this degree should be evident, because as long as each degree's quantity is not specified, the degree is also not known ${ }^{68}$ because the naming of the degrees is according to the strength and weakness of their effects, just as was mentioned in the first preliminary. Because, for example, if there were a strong increase in the quantities used of something we say to be hot in the first degree, and if it is able to leave a trace like the effect of something in the fourth degree, then the first degree is not specified and known. Specifically, if just a little of something that is hot in the fourth degree is used, it appears like the effect of [something] in the first degree. But we can also say that the first degree is hot. Thus in order to say that a compound at some degree is hot, it is necessary to know the measure of the degree.

The author of the Múcaz said without exception that the quantity of this simple [medicine]

$3 a$

being used is this measure of the quantity of the degree. ${ }^{69}$ But Isaac al-Isrā'ìlì mentioned, "I did not see whoever mentioned this subject." It is from a European physician who was not well-known, called Arnaldo da Villanova. This he said, their

67 This first preliminary is spelled out in a summary fashion in Ibn al-Nafīs (ed. 'Abd al-Karīm al-'Azabāwī), al-Mūjaz fĩ al-țibb, (Cairo: Wizārat al-awqāf, 1986): 77.

68 Mūsā Cālinūs has introduced the third preliminary as an attempt to resolve the problem of how the effect alone will not indicate clearly the degree of the simple medication. One must know the quantity of the medicine.

69 This may mean that there was some connection between the amount of a simple medicine that was used and the extent of its effect. The Mücaz (77) related the degrees to the relative intensity of the simple medicine, but did not explore whether a high quantity of a simple medicine in the first degree might be harmful. The rest of the paragraph argues that a greater quantity of a medicine of a lower degree might have the effect of a lesser quantity of a medicine at a higher degree. 
primaries'70 amount in the first degree was three drams, and the second degree's is two, and the third degree's was one and a half, and the fourth degree's was one. ${ }^{71}$ He believed in and depended upon this opinion. In this way, for example, a compound that we say is hot in the second, its meaning at the time is this: Two drams from some balanced mixture should manifest the effect of something in the second degree, just as was mentioned in the first preliminary. And if we deduce from the rules that we shall mention that a compound is hot in the fourth degree, it is necessary to know that from this compound's being hot in the fourth degree, one dram of a balanced mixture suffices at a certain times. The same goes for the rest of the degrees.

But Muhammad Ibn Rushd, the author of the Mūcaz, and the rest of the scholars also did not take this position because all of the medicines that are at one degree do not have the same measures. For example, it is necessary to consume twenty drams of honey that are hot in the second degree. If we make it into a compound with two drams of sandalwood that are cold in the second degree, it then becomes a compound at equilibrium. But if two drams of honey were taken, then it does not remain in equilibrium with sandalwood's cold. Therefore, each species has a particular measure. I and some of the scholars of our time also became perplexed by this thing until, with the help of Allah, I found it in a composition of Ibn Zuhr, ${ }^{72}$ and from that book I copied this book's simple medicines and named it The Measure of Medicines. But whatever compound's quantities he did not write down, then according to this method the medicine's quantity needs to be known either experimentally or by making a ratio to the simples. But on account of this ratio being very complicated, and due to how the above-mentioned Arnaldo's method is also more truthful, and on account of how a mistake does not occur on account of this method's error, we also work according to that in this composition.

70 I am reading اول as 'primary'.

71 The most likely source, as the wording parallels the Ottoman Turkish, for this report is Joshua Lorqi, Gerem ha-ma'alot, Austrian National Library Hebrew 45, 89b. For a possible Latin source, see Arnaldo de Villanova (ed., intro., and comm. Michael R. McVaugh): Opera medica omnia. 2, Aphorismi de gradibus (Granada and Barcelona, 1975): 244. In his commentary on Arnaldo's De Gradibus, McVaugh quoted from another of Arnaldo's works, the Speculum medicine: "De qua due regule collecte sunt per experientiam a sapientibus, et prima est de dosibus complexionatorum in quantum absolutam habent rationem alternatium medicinarum; secunda vero de dosibus in quantum habent manifestam nutriendi potentiam. De absolute vero alterantibus est compertum in temperata regione atque in corpore temperato quod prima dosis complexionatorum in quarto gradu est 31 , in tertio vero 31 et $1 / 2$, in secundo vero 32 , in primo vero 33 ; in temperato vero ut temperatum [Ed. intemperatum] est non convenit certa dosis." The second set of numbers $\left(1,1 \frac{1}{2}, 2,3\right)$ is most important for the comparison with the Ottoman text. McVaugh noted that the same rule exists in Arnaldo's Antidotarium.

This is most likely the epistle dedicated to the padişah. See note 192. 
The fourth preliminary is this: To know each degree's parts

$3 b$

means [to know] their qualities. [Bernard de] Gordon and most scholars presumed that the ratio of the parts of a quality is according to the following ratio of doubling. ${ }^{73}$ I mean, being hot in the first degree, for example, means that there are two parts of heat, because one part [of heat] and a cold part can return to equilibrium because the second part of heat is dominant until it is hot in the first [degree]. And if it has four hot parts, then it is hot in the second degree. And if [it has] eight [hot parts], [it is hot] in the third. If it has 16 hot parts, then it is hot in the fourth. But this opinion is in the thing in itself (nafs-i amr) ${ }^{74}$, and, according to Muhammad ibn Rushd and the author of the $M \bar{u} c a z^{75}$, it is a mistake because an increase in the degree is derived from an increase in the parts. If the increase of the degree is equal, then the increase of the parts must also be equal. ${ }^{76}$ I mean, for example, that if a little of this medicine's heat is felt in the body, then we say that heat in the first [degree belongs] to it, and that if two of these measures of heat are felt, then it is hot in the second. And if three of these measures [are felt], then it is hot in the third. And if four of these measures [are felt], it is hot in the fourth.

The rest of the qualities follow this analogy. Therefore, the increase of the degrees follows the ratio of the natural numbers. For example, something being cold in the first degree means that it has one dominant part of cold. I mean that to this simple medicine's one part of heat, there are two parts cold, one of them being together [with the heat] and the other one being dominant so that, therefore, it was cold in the first degree. The second [degree] has two dominant [parts], and the third three. If the fourth degree should comprise four dominant [parts], then being hot in the first degree, for example, means that there are two parts of heat, that something hot in the second has three [parts] of heat, that something in the third degree has four [parts of heat], and something in the fourth degree has five

74 On nafs-i amr, see İhsan Fazlığlu, "Between Reality and Mentality- Fifteenth Century Mathematics and Natural Philosophy Reconsidered," in Nazariyat I, (2014): 1-39, at 24-30 and Recep Duran, "Nefsü'l-Emr Risaleleri," in Ankara Üniversitesi Dil ve Tarih-Coğrafya Fakültesi Araştırma Dergisi XIV, (1992): 97-106

75 See Nahyan Fancy, Science and Religion in Mamluk Egypt: Ibn al-Nafis, pulmonary transit and bodily resurrection (London: Routledge, 2013), 116-20. Here, Fancy pointed out that Ibn al-Nafis may not have written the Mūcaz. See also Fancy, "Medical Commentaries: A Preliminary Examination of Ibn al-Nafīs's Shurūḥ, the Mūjaz, and Subsequent Commentaries on the Mūjaz" Oriens XLI, (2013): 525-45, at 535. Fancy "raises some concerns with regards to the authenticity of the Müjaz and its attribution to Ibn al-Nafīs." 
[parts of heat]. In general, the measure [of the dominant quality] is always one in excess of the name of the degree. This is how we talk, I mean, because supposing one part in excess is always easier to imagine and in the thing in itself (nafs-i amr), on account of how all created things are compounded from opposing elements. As for your wish to suppose that, for example, something hot in the first degree has, for example, one part cold, either in this supposition we suppose without exception a hot part for it, and the second has two parts of it, and the third three and the fourth, four; or without exception there should be no hot parts. The two ways'

$4 \mathrm{a}$

result is one, because, for example, if there is something we say is hot in the first degree, for if we give two hot parts and one cold, then one hot part remains. And if, from the beginning, we gave absolutely one part hot and not a cold part, then in this there is also a single part. Therefore, these ways are one.

But we say that the first scholars' opinion, although it might not be a true supposition in the thing in itself (nafs-i amr), it is the rationale for finding an easier way to arrive at the true result, just as the orbs of the epicycle and the eccentric are the rationale for finding the true positions of the planets, although they themselves are an impossible and deceptive supposition, just as Ptolemy himself mentioned. Therefore Arnaldo and Gordon worked according to this. Rather, alKindi's ${ }^{77}$ supposition is that being the pretext for arriving quickly at the truth of the matter is akin to [being] the reason. These 16 parts that they supposed are like the fractions of the calculation ${ }^{78}$, for with these is the rationale for finding, more correctly and more easily, the numerical ratio. In general, there is no more precise and easier way than this that we might arrive from the truth at the ratio. Therefore, we should work according to this. Some individuals, on account of their seeing the correctness of this way, wrote epistles to establish it because they presumed that for a true effect the middle term [of a syllogism] is true, just as some individuals wrote epistles to establish the orb of the epicycle and the eccentric. ${ }^{79}$ But they erred

77 This spelling of al-Kindī (viz. al-Qindī) could be evidence that either Mūsā Câlīnūs and/or the copyist of the MS had access to Latin texts as al-Kindī is spelled 'Alquindus' in Arnaldo da Villanova's Aphorismi de Gradibus [see, e.g., Arnaldo de Villanova, (ed., intro. and comm. McVaugh), De Gradibus, 181]. It could also be a spelling convention.

78 Mūsā Cālinnūs may be saying that the proportions of simple medicines in compounds may not always fit the ratios perfectly.

79 Cf. Arnaldo de Villanova, (ed., intro. and comm. McVaugh), De Gradibus, 176. 
because Ptolemy himself, who is the head and master of the craft, confirmed in his Almagest that this proposition is impossible. ${ }^{80}$

The fifth preliminary is this: A common and shared weakness ${ }^{81}$ weakens the stronger, and also the opposite, meaning that strength accrues to the weaker from the stronger. ${ }^{82}$ Thus the degrees of medicines of a single quality might vary, and likewise, when they are combined the degree of the greater degree with the lesser degree is less. For example, if we combine something hot in the fourth degree with something hot in the first, their quantities' being related in value makes the thing that is hot in the fourth degree descend from its degree; so it is a decrease. All of the scholars say so, that is, if it is one measure of decrease.

$4 \mathrm{~b}$

And this is also apparent by observation, because if we mix tepid water with extremely hot water, then its heat always decreases. But at what degree or by what measure does it decrease? ${ }^{83}$ There are differences among the scholars because some of the physicians presumed that, for example, if we mix a weight-measure of something cold in the first degree with a weight-measure of something cold in the third degree, a compound cold in the second degree is the result. But this opinion is a mistake because if heat causes heat to decline this amount, it would be necessary that it be hot in the first and cold in the third. It would have reduced it to the first degree. And if that were the case, it would be necessary that [something] hot in the second and cold in the third to remain equilibrate. But it is not necessary to know, in our way, what amount reduces it because al-Kindī's method is a general rule. If it is necessary that its qualities be different but its degrees agree, it is also necessary

80 Elsewhere, Mūsā Cālīnūs held that Ptolemy had accepted the hypotheses of the eccentric and epicycle which were, to Mūsā Cālīnūs, philosophically objectionable. See Robert Morrison: "An Astronomical Treatise by Mūsā Jālīnūs alias Moses Galeano," Aleph: Historical Studies in Science and Judaism X/2, (2011): 385-413, at 396 and 404. Mūsā Cālīnūs may be saying that, according to his reading of The Almagest, the eccentric and the epicycle served as devices to calculate planetary positions, but were not necessarily true, either externally or in nafs-i amr. Or, Mūsā Cālīnūs could be referring to Book Nine of The Almagest where Ptolemy expressed his reservations about his own models. I thank Jamil Ragep of McGill University for his suggestion about Book Nine of The Almagest.

81 See Léon Gauthier, Antécédents gréco-arabes de la psychophysique, (Beirut: Imprimerie Catholique, 1938): 31 and 74 (in the Arabic).

82 Cf. Ibn Rushd (ed. J.M. Fórneas Besteiro and C. Alvarez de Morales), Kitāab al-Kulliyyāt fï al-țibb, (Madrid: Consejo Superior de Investigaciones Científicas ; Granada : Escuela de Estudios Arabes, 1987): 387.

83 Cf. Ibn Rushd's Kulliyyāt as cited and translated by Langermann, “Another Andalusian Revolt," 354: "This law (al- qānūn), I mean that a drug having less heat will reduce the heat of [a drug possessing] more [heat] was confirmed (yușahhihuhu) by Galen. He took as evidence [the case of] hot and tepid water. When the hot is mixed with the tepid, its heat is necessarily reduced." This follows Ibn Rushd's Kulliyyāt, 383-4. 
that its degrees be different [after being mixed].$^{84}$ But its qualities should agree [after being mixed] if it is necessary that in two of them together they [the qualities] are different, and if it is also necessary that they should also be different in their quantities. The rule is particular and general.

But if a weak medicine is equilibrate, I mean that if its non-equilibrate simple medicines are assembled into an equilibrated medicine, then the rule for it is also this: The ratio of the quality to the quality must equal the ratio of the quantity to the quantity. I mean that whatever ratio there is of a quantity of an equilibrate medicine to a quantity of a non-equilibrate medicine, on account of this it is necessary to combine the quality's parts on the likes of this ratio. For example, if the measure is twice a non-equilibrate, then because of this we take two parts of something hot and two of something cold and two of something moist and two of something dry. We assemble its cold [parts] with the cold and its hot [parts] with the hot. And if the measure is five non-equilibrate [parts], on account of this we take five parts cold and five [parts] hot. Likewise if the measure is one-third [of a part] of an non-equilibrate medicine, [we take] a third of a part of something hot or cold. ${ }^{85}$ And if it is a fourth, then a fourth, and likewise in the rest of the ratios. It should be known that according to this preliminary, one sophistical objection is necessary. I mean that if this rule ${ }^{86}$ is true, it would be necessary that honey might be useful for a burning fever, the heat of which is hot at its extreme because its heat is less than the heat of a burning fever. But the necessary consequence is clearly null because it [the honey] is extremely harmful to the fever; however, the premise is also null. We say in response that honey, inasmuch as it is hot in the second degree, is, without a doubt, useful to the fever

$5 a$

with respect to that [part] of it that is fever. And let it be extremely harmful accidentally, I mean on account of its being quickly inverted to yellow bile, just as melon and the rest of the fruits, being particularly cold and moist, are essentially useful to fevers inasmuch as they are fevers. But they are accidentally harmful because they quickly putrefy.

This argument is counter-intuitive, and Mūsā Cālīnūs seems to reject it on the last page of the text. Mūsā Cālīnūs is saying that you would not add two parts of cold, hot, wet, and dry. Rather, you would add the parts that are necessary to make the compound equilibrate. Or, you would add the cold/hot/ moist/dry parts in order to make the compound non-equilibrate.

Namely, that the ratio of the quantity to the quantity is the ratio of the quality to the quality. 
The third chapter. In order to know the compound's degree for every measure, for example, for every single dram, he [the physician] should give parts from the quality suitable to this degree and to its quality's species. I mean, the first degree has two parts of heat or of cold, or of the other qualities according to the medicine's quality. And to the second [degree] four [parts], and to the third eight, and to the fourth sixteen, and to each dram of a quality of a simple [medicine] one part of an opposite quality is to be given. We should assemble the qualities from each species and consider the parts assembled from each quality. They have their ratios to them with their opposite parts. Then, we look at this ratio to determine which ratio of the parts of a degree it resembles, and then this quality is of this degree. For example, we wanted to know theriac's four degrees:

\begin{tabular}{|c|c|c|c|c|c|}
\hline $\begin{array}{l}\text { The names of the } \\
\text { medicines }\end{array}$ & hot parts & cold parts & dry parts & $\begin{array}{l}\text { moist } \\
\text { parts }\end{array}$ & drams \\
\hline Aristolochia $^{87}$ & 60 & 5 & 40 & 5 & 5 \\
\hline Gentiana & 40 & 5 & 60 & 5 & 5 \\
\hline Laurel seed $^{88}$ & 40 & 5 & 40 & 5 & 5 \\
\hline Myrrh & 60 & 5 & 60 & 5 & 5 \\
\hline Honey & 240 & 60 & 240 & 60 & 60 \\
\hline Total & 440 & 80 & 440 & $100^{89}$ & $100^{90}$ \\
\hline
\end{tabular}

We wrote the medicines on one side, and then each quality's parts and the weight of drams [on the other]. Then we assembled, separately, the qualities from each species. We saw that the parts of heat are on the measure of five parts of cold ${ }^{91}$, since if they [the parts of heat] were on the measure of four parts of cold, its heat would be at the second degree. Presently, approximately, the parts of heat are approximately on the measure of six parts of cold, and thus is in the middle of the third degree. I mean one and a half drams are sufficient. And because the parts of dryness are on the measure of four parts of cold, it will increase by a little and is, therefore, dry in the beginning of the third degree. I mean two drams are clearly sufficient. with a Study of its Materia Medica by Martin Levey (Madison, Milwaukee, and London: The University of Wisconsin Press, 1966): 273.

88 Kindi, Formulary, 308-9.

89 The moist parts do not add up.

90 The drams do not add up.

91 That is, each part of heat equals five parts cold. There is a kırk ziyadda'dir in a supra-linear insertion. Including the forty helps the math come out correctly because $\left(80^{*} 5\right)+40$ is 440 . 
A compound's degree in the philosophers' qualities

\begin{tabular}{|c|c|c|c|c|c|c|}
\hline $\begin{array}{l}\text { The names of the } \\
\text { medicines }\end{array}$ & $\begin{array}{c}\text { their } \\
\text { natures }\end{array}$ & $\begin{array}{c}\text { their } \\
\text { weights }\end{array}$ & hot parts & cold parts & dry parts & $\begin{array}{l}\text { moist } \\
\text { parts }\end{array}$ \\
\hline Pepper & $4 \mathrm{~h} / \mathrm{d}$ & 10 drams & 160 & 10 & 10 & 160 \\
\hline Long pepper ${ }^{92}$ & $3 \mathrm{~h} / 2 \mathrm{~d}$ & 10 & 80 & 10 & 10 & 40 \\
\hline Clove $^{93}$ & $3 \mathrm{~h} / \mathrm{d}$ & 10 & 80 & 10 & 10 & 80 \\
\hline Ginger & $2 \mathrm{~h} / 1 \mathrm{~d}$ & 10 & 40 & 10 & 10 & 20 \\
\hline Emblic Myrobalan ${ }^{94}$ & $1 c$ & 10 & 10 & 20 & 10 & 20 \\
\hline Beleric $^{95}$ & $1 c / 2 d$ & 10 & 10 & 20 & 10 & 40 \\
\hline Camomile $^{96}$ & $2 \mathrm{~h} / \mathrm{d}$ & 5 & 20 & 5 & 5 & 20 \\
\hline Root (așl) of camomile & $2 \mathrm{~h} / \mathrm{d}$ & 10 & 40 & 10 & 10 & 40 \\
\hline Cinnamon $^{97}$ & $2 \mathrm{~h} / \mathrm{d}$ & 10 & 80 & 10 & 10 & 80 \\
\hline $\begin{array}{l}\text { Large species of } \\
\text { swallow wort }\end{array}$ & $2 \mathrm{~h} / \mathrm{d}$ & 10 & 40 & 10 & 10 & 40 \\
\hline Fox testicle & $1 \mathrm{~h} / \mathrm{m}$ & 10 & 20 & 10 & 20 & 10 \\
\hline Red bloom raisins & $2 \mathrm{~h} / \mathrm{d}$ & 30 & 120 & 30 & 30 & 120 \\
\hline Round Aristolochia $^{98}$ & $2 \mathrm{~h} / 3 \mathrm{~d}$ & 10 & 40 & 10 & 10 & 80 \\
\hline Coconut & $1 \mathrm{~h} / \mathrm{d}$ & 10 & 20 & 10 & 10 & 20 \\
\hline Pepperwort $^{99}$ & $2 \mathrm{~h} / \mathrm{d}$ & 10 & 80 & 10 & 10 & 80 \\
\hline Pine $^{100}$ & $1 \mathrm{~h} / \mathrm{d}$ & 30 & 60 & 30 & 30 & 60 \\
\hline Honey & $2 \mathrm{~h} / \mathrm{d}$ & 555 & 2220 & 555 & 555 & 2220 \\
\hline Total & \multicolumn{2}{|c|}{ end of $2 h / d$} & 2920 & 760 & 760 & 3130 \\
\hline
\end{tabular}

92 Kindī, Formulary, 266-7.

93 Kindi, Formulary, 315-6.

94 Kindī, Formulary, 235.

95 Ibn al-Tilmìdh, Dispensary, 324.

96 Kindī, Formulary, 239.

97 Kindi, Formulary, 265-6.

98 Kindī, Formulary, 273-4.

99 Kindi, Formulary, 296-7.

100 Kindi, Formulary, 299-300. 
$6 a$

\begin{tabular}{|c|c|c|c|c|c|c|}
\hline $\begin{array}{l}\text { The parts of the paste } \\
\text { of Falūniyā }\end{array}$ & $\begin{array}{l}\text { weight in } \\
\text { drams }\end{array}$ & nature & hot parts & cold parts & moist parts & dry parts \\
\hline Saffron $^{101}$ & 5 & $2 \mathrm{~h} / 1 \mathrm{~d}$ & 20 & 5 & 5 & 10 \\
\hline Pepper & 20 & $3 \mathrm{~h} / \mathrm{d}$ & 320 & 20 & 20 & 320 \\
\hline Henbane seed ${ }^{102}$ & 20 & $2 \mathrm{~h} / \mathrm{d}$ & 80 & 20 & 20 & 80 \\
\hline Opium & 10 & $4 \mathrm{~h} / \mathrm{d}$ & 160 & 10 & 10 & 16 \\
\hline Rock parsley ${ }^{103}$ & 1 & $3 \mathrm{~h} / \mathrm{d}$ & 8 & 1 & 1 & 8 \\
\hline Euphorbium $^{104}$ & 1 & $4 \mathrm{~h} / \mathrm{d}$ & 16 & 1 & 1 & 16 \\
\hline $\begin{array}{l}\text { Oil of balm of } \\
\text { Gilead }^{105}\end{array}$ & 5 & $2 \mathrm{~h} / \mathrm{d}$ & 40 & 5 & 5 & 40 \\
\hline Honey & 186 & & 744 & 186 & 186 & 744 \\
\hline Total & \multicolumn{2}{|c|}{ Beg. of 3h/d } & 1388 & 248 & 248 & 1234 \\
\hline
\end{tabular}

\begin{tabular}{|c|c|c|c|c|c|c|}
\hline $\begin{array}{l}\text { The parts of a } \\
\text { compound electuary }{ }^{106}\end{array}$ & $\begin{array}{l}\text { weight in } \\
\text { drams }\end{array}$ & nature & hot parts & cold parts & moist parts & dry parts \\
\hline Mastic & 2 & $2 \mathrm{~h} / \mathrm{d}$ & 8 & 2 & 2 & 8 \\
\hline Saffron & 2 & $2 \mathrm{~h} / 1 \mathrm{~d}$ & 8 & 2 & 2 & 4 \\
\hline Nard & 2 & $1 \mathrm{~h} / 2 \mathrm{~d}$ & 8 & 2 & 2 & 8 \\
\hline Seed of balsam & 2 & $2 \mathrm{~h} / \mathrm{d}$ & 8 & 2 & 2 & 8 \\
\hline Asarum $^{107}$ & 2 & $3 \mathrm{~h} / \mathrm{d}$ & 16 & 2 & 2 & 16 \\
\hline Balsam of ban tree & 2 & $3 \mathrm{~h} / \mathrm{d}$ & 16 & 2 & 2 & 16 \\
\hline Cinnamon & 2 & $3 \mathrm{~h} / \mathrm{d}$ & 16 & 2 & 2 & 16 \\
\hline Lemon grass $^{108}$ & 2 & $3 \mathrm{~h} / \mathrm{d}$ & 8 & 2 & 2 & 8 \\
\hline Aloe plant ${ }^{109}$ & 12 & $3 \mathrm{~h} / \mathrm{d}$ & 128 & 16 & 16 & 128 \\
\hline Honey & 24 & $2 \mathrm{~h} / \mathrm{d}$ & 96 & 24 & 24 & 96 \\
\hline Total & \multicolumn{2}{|c|}{ Beg. of $3 h / d$} & 312 & 56 & 56 & 308 \\
\hline
\end{tabular}

101 Kindī, Formulary, 275-6.

102 Kindī, Formulary, 246.

103 Or parsley seed or a kind of mushroom.

104 Kindī, Formulary, 311 (as furbiyūn). Steingass (A Comprehensive Persian-English Dictionary, 921) vocalized it as furfiyūn and farfayūn.

105 Kindi, Formulary, 245.

106 Kindi, Formulary, 238.

107 Kindī, Formulary, 227.

108 Kindi, Formulary, 225-6.

109 Kindī, Formulary, 297. 
$6 \mathrm{~b}$

\begin{tabular}{|c|c|c|c|c|c|c|}
\hline $\begin{array}{l}\text { The parts of a narcotic } \\
\text { electuary }\end{array}$ & $\begin{array}{l}\text { weight in } \\
\text { drams }\end{array}$ & nature & hot parts & cold parts & $\begin{array}{l}\text { moist } \\
\text { parts }\end{array}$ & dry parts \\
\hline White pepper & 20 & $4 \mathrm{~h} / \mathrm{d}$ & 320 & 20 & 20 & 320 \\
\hline Black pepper & 20 & $4 \mathrm{~h} / \mathrm{d}$ & 320 & 20 & 20 & 320 \\
\hline Henbane seed & 20 & $2 \mathrm{c} / \mathrm{d}$ & 20 & 80 & 20 & 80 \\
\hline Opium & 10 & $4 c / d$ & 10 & 160 & 10 & 160 \\
\hline Saffron & 7 & $2 \mathrm{~h} / 1 \mathrm{~d}$ & 28 & 7 & 7 & 14 \\
\hline Nard & 1.5 & $1 \mathrm{~h} / 2 \mathrm{~d}$ & 3 & 1.5 & 1.5 & 6 \\
\hline Ephorbium & 1.5 & $4 \mathrm{~h} / \mathrm{d}$ & 34 & 1.5 & 1.5 & 24 \\
\hline Beaver testicle $^{110}$ & 1.5 & $3 \mathrm{~h} / \mathrm{d}$ & 12 & 1.5 & 1.5 & 12 \\
\hline Pyrethum $^{111}$ & 1.5 & $3 \mathrm{~h} / \mathrm{d}$ & 12 & 1.5 & 1.5 & 12 \\
\hline Honey & 249 & $2 \mathrm{~h} / \mathrm{d}$ & 996 & 249 & 249 & 996 \\
\hline Total & \multicolumn{2}{|c|}{$2 h / 3 d$} & 1755 & $533^{112}$ & 332 & 1944 \\
\hline
\end{tabular}

\begin{tabular}{|c|c|c|c|c|c|c|}
\hline $\begin{array}{l}\text { The parts of a medicine } \\
\text { taken dry }{ }^{113}\end{array}$ & $\begin{array}{l}\text { weight in } \\
\text { drams }\end{array}$ & nature & hot parts & cold parts & $\begin{array}{l}\text { moist } \\
\text { parts }\end{array}$ & $\begin{array}{c}\text { dry } \\
\text { parts }\end{array}$ \\
\hline Scammony & 7.5 & $3 \mathrm{~h} / \mathrm{d}$ & 60 & 7.5 & 7.5 & 60 \\
\hline White pepper & 4.5 & $4 \mathrm{~h} / \mathrm{d}$ & 70 & 4.5 & 4.5 & 70 \\
\hline Cardamom $^{114}$ & 4.5 & $3 \mathrm{~h} / \mathrm{d}$ & 36 & 4.5 & 4.5 & 36 \\
\hline Ginger & 1.5 & $3 \mathrm{~h} / \mathrm{d}$ & 12 & 1.5 & 1.5 & 12 \\
\hline Cinnamon & 1.5 & $3 \mathrm{~h} / \mathrm{d}$ & 12 & 1.5 & 1.5 & 12 \\
\hline Mace & 1.5 & $2 \mathrm{~h} / \mathrm{d}$ & 6 & 1.5 & 1.5 & 6 \\
\hline Clove & 1.5 & $3 \mathrm{~h} / \mathrm{d}$ & 12 & 1.5 & 1.5 & 12 \\
\hline Nutmeg $^{115}$ & 1.5 & $2 \mathrm{~h} / \mathrm{d}$ & 6 & 1.5 & 1.5 & 6 \\
\hline Emblic myrobalan & 1.5 & $1 c / d$ & 1.5 & 3 & 1.5 & 3 \\
\hline White sugar $^{116}$ & 50 & $1 \mathrm{~h} / \mathrm{d}$ & 100 & 50 & 100 & 50 \\
\hline Honey & 105 & $2 \mathrm{~h} / \mathrm{d}$ & 420 & 105 & 105 & 420 \\
\hline Total & \multicolumn{2}{|c|}{ end of $2 h / \mathrm{mid}-1 \mathrm{~d}$} & 735 & 182 & 230 & 687 \\
\hline
\end{tabular}

110 Kindī, Formulary, 254.

111 Kindi, Formulary, 301-2.

112 The sum is actually 542 .

113 It's a medicine (safüf) taken dry, not mixed with water (as in the case of an electuary). See Martin Levey, Early Arabic Pharmacology, (Leiden: Brill, 1973,1981): 81.

114 Kindi, Formulary, 313-4.

115 Kindī, Formulary, 256.

116 Kindi, Formulary, 284. 


\begin{tabular}{|c|c|c|c|c|c|c|}
\hline $\begin{array}{l}\text { The parts of a paste of an } \\
\text { electuary } 117\end{array}$ & $\begin{array}{l}\text { weight in } \\
\text { drams }\end{array}$ & nature & $\begin{array}{l}\text { hot } \\
\text { parts }\end{array}$ & $\begin{array}{l}\text { cold } \\
\text { parts }\end{array}$ & $\begin{array}{l}\text { moist } \\
\text { parts }\end{array}$ & $\begin{array}{c}\text { dry } \\
\text { parts }\end{array}$ \\
\hline Red rose $\mathrm{e}^{118}$ & 6 & $1 c / 2 d$ & 6 & 12 & 6 & 24 \\
\hline Cyperus longus $^{119}$ & 5 & $3 \mathrm{~h} / \mathrm{d}$ & 40 & 5 & 5 & 40 \\
\hline Clove & 3 & $3 \mathrm{~h} / \mathrm{d}$ & 24 & 3 & 3 & 24 \\
\hline Mastic & 3 & $2 \mathrm{~h} / \mathrm{d}$ & 12 & 3 & 3 & 12 \\
\hline Nard & 3 & $1 \mathrm{~h} / 2 \mathrm{~d}$ & 6 & 3 & 3 & 12 \\
\hline Asarum & 3 & $3 \mathrm{~h} / \mathrm{d}$ & 24 & 3 & 3 & 24 \\
\hline Cinnamon (inferior) ${ }^{120}$ & 2 & $3 \mathrm{~h} / \mathrm{d}$ & 16 & 2 & 2 & 16 \\
\hline Yew $^{121}$ & 2 & beg3h/d & 11 & 2 & 2 & 11 \\
\hline Saffron & 2 & $2 \mathrm{~h} / 1 \mathrm{~d}$ & 8 & 2 & 2 & 4 \\
\hline Mace & 2 & $2 \mathrm{~h} / \mathrm{d}$ & 8 & 2 & 2 & 8 \\
\hline Cardamom & 2 & $3 \mathrm{~h} / \mathrm{d}$ & 16 & 2 & 2 & 16 \\
\hline Lesser cardamom (hayl) & 2 & $3 \mathrm{~h} / \mathrm{d}$ & 16 & 2 & 2 & 16 \\
\hline Nutmeg & 2 & $2 \mathrm{~h} / \mathrm{d}$ & 8 & 2 & 2 & 8 \\
\hline Emblic myrobalan & 100 & $1 c / d$ & 100 & 100 & 100 & 200 \\
\hline Sugar candy $(f a \bar{n} n \bar{\imath} \underline{z})^{122}$ & 200 & $1 \mathrm{~h} / \mathrm{d}$ & 400 & 400 & 400 & 200 \\
\hline Total & \multicolumn{2}{|c|}{$\operatorname{mid}-1 h / d$} & 695 & 443 & 537 & 615 \\
\hline
\end{tabular}

$7 b$

\begin{tabular}{|c|c|c|c|c|c|c|}
\hline The parts of the theriac of Mithridates ${ }^{123}$ & $\begin{array}{c}\text { weight in } \\
\text { drams }\end{array}$ & nature & $\begin{array}{c}\text { hot } \\
\text { parts }\end{array}$ & $\begin{array}{c}\text { cold } \\
\text { parts }\end{array}$ & $\begin{array}{l}\text { moist } \\
\text { parts }\end{array}$ & $\begin{array}{c}\text { dry } \\
\text { parts }\end{array}$ \\
\hline Myrrh & 10 & $2 \mathrm{~h} / \mathrm{d}$ & 40 & 10 & 10 & 40 \\
\hline Saffron & 10 & $1 \mathrm{~h} / \mathrm{d}$ & 20 & 10 & 10 & 20 \\
\hline Agaric $^{124}$ & 10 & $2 \mathrm{~h} / \mathrm{d}$ & 40 & 10 & 10 & 40 \\
\hline
\end{tabular}

118 Kindi, Formulary, 344-5. Also, see the similar recipe in Kindī, Formulary, 34.

119 Kindī, Formulary, 282.

120 Kindi, Formulary, 315.

121 Kindi, Formulary, 274. Steingass (Comprehensive Dictionary, 616) states that zarnab is an odoriferous plant and/or saffron.

122 Kindi, Formulary, 312.

123 Even in Antiquity, there was no single recipe for the theriac of Mithridates. See Laurence Totelin, "Mithradates' Antidote: A Pharmacological Ghost," in Early Science and Medicine IX, (2004): 1-19. The recipe for the theriac of Mithradates in 'Alī b. Sahl al-Ṭabarī (ed. M. Z. Siddiqi), Firdaws al-hikma fí alțibb, (Berlin: The E.G.W. Gibb Memorial Trust and Sonnen-Druckerei, 1928): 462 is similar, but not exactly the same. The recipe of Sābūr ibn Sahl (ed., trans., and notes Oliver Kahl), Sābūr ibn Sahl's Dispensary in the Recensions of the 'Adudi Hospital, (Boston: Brill, 2008): 174 is also quite similar.

124 Kindī, Formulary, 309. 


\begin{tabular}{|c|c|c|c|c|c|c|}
\hline Ginger & 10 & $2 \mathrm{~h} / 3 \mathrm{~d}$ & 80 & 10 & 10 & 80 \\
\hline Cinnamon & 10 & $3 \mathrm{~h} / \mathrm{d}$ & 80 & 10 & 10 & 80 \\
\hline Gum of Nabataeans ${ }^{125}$ & 10 & $3 \mathrm{~h} / \mathrm{d}$ & 80 & 10 & 10 & 80 \\
\hline Gum tragacanth ${ }^{126}$ & 10 & $1 \mathrm{~h} / \mathrm{d}$ & 20 & 10 & 10 & 20 \\
\hline Indian nard ${ }^{127}$ & 8 & $1 \mathrm{~h} / \mathrm{d}$ & 16 & 8 & 8 & 16 \\
\hline Oriental frankincense ${ }^{128}$ & 8 & $2 \mathrm{~h} / 1 \mathrm{~d}$ & 16 & 8 & 8 & 16 \\
\hline White mustard $^{129}$ & 8 & $4 \mathrm{~h} / \mathrm{d}$ & 128 & 8 & 8 & 128 \\
\hline Tall palms of balsam & 8 & $2 \mathrm{~h} / \mathrm{d}$ & 32 & 8 & 8 & 32 \\
\hline French lavender & 8 & $1 \mathrm{~h} / 2 \mathrm{~d}$ & 12 & 8 & 8 & 32 \\
\hline Lemon grass & 8 & $2 \mathrm{~h} / \mathrm{d}$ & 32 & 8 & 8 & 32 \\
\hline Costus ${ }^{130}$ & 8 & $3 \mathrm{~h} / 2 \mathrm{~d}$ & 64 & 8 & 8 & 32 \\
\hline Assafoetida & 8 & $3 \mathrm{~h} / \mathrm{d}$ & 64 & 8 & 8 & 64 \\
\hline Ground-pine $^{131}$ & 8 & $3 \mathrm{~h} / \mathrm{d}$ & 64 & 8 & 8 & 64 \\
\hline Galbanum $^{132}$ & 8 & $3 \mathrm{~h} / \mathrm{d}$ & 32 & 8 & 8 & 64 \\
\hline (Pine) Resin ${ }^{133}$ & 8 & $3 \mathrm{~h} / 1 \mathrm{~d}$ & 64 & 8 & 8 & 32 \\
\hline Long pepper ${ }^{134}$ & 8 & $3 \mathrm{~h} / 2 \mathrm{~d}$ & 64 & 8 & 8 & 32 \\
\hline Salsify ${ }^{135}$ & 8 & $1 c / 2 d$ & 8 & 16 & 8 & 32 \\
\hline Beaver testicle & 8 & $3 \mathrm{~h} / \mathrm{d}$ & 64 & 8 & 8 & 64 \\
\hline Opopanax $^{136}$ & 8 & $2 \mathrm{~h} / \mathrm{d}$ & 32 & 8 & 8 & 32 \\
\hline Indian laurel $^{137}$ & 8 & $2 \mathrm{~h} / \mathrm{d}$ & 32 & 8 & 8 & 32 \\
\hline Liquid storax $^{138}$ & 8 & $1 \mathrm{~h} / 2 \mathrm{~d}$ & 32 & 8 & 8 & 32 \\
\hline Cinnamon bark $^{139}$ & 7 & $3 \mathrm{~h} / \mathrm{d}$ & 64 & 7 & 7 & 64 \\
\hline White pepper & 7 & $4 \mathrm{~h} / \mathrm{d}$ & 128 & 7 & 7 & 128 \\
\hline Black pepper & 7 & $4 \mathrm{~h} / \mathrm{d}$ & 128 & 7 & 7 & 128 \\
\hline Colchicum & 7 & $2 \mathrm{~h} / \mathrm{d}$ & 32 & 7 & 7 & 32 \\
\hline
\end{tabular}

125 Kindī, Formulary, 306. There is a marginal notation in the MS correcting 'ilk al-anbāt to 'ilk al-buțm, but Levey writes that they are the same thing.

126 Kindi, Formulary, 323.

127 Kindī, Formulary, 286-7. The MS has sunbul hindī, but Kindī gives sunbul al-țīb for Indian nard.

128 Sābūr b. Sahl, Dispensary, 242.

129 Kindi, Formulary, 264.

130 Kindi, Formulary, 316.

131 Steingass, Comprehensive Dictionary, 1047.

132 Kindi, Formulary, 319.

133 Kindī, Formulary, 269.

134 Kindī, Formulary, 266-7.

135 The text has 'uṣārat al-hayūfasțīdās, but according to the Känūn, this simple is the same as lihyat al-tays.

136 Kindi, Formulary, 254-5. See also Steingass, Comprehensive Dictionary, 854.

137 Oliver Kahl, The Dispensatory of Ibn at-Tilmīd, (Leiden and Boston: Brill, 2007): 328. Kindī (Formulary, 279) suggests 'spikenard'.

138 Kindī, Formulary, 338.

139 Or Chinese cinnamon tree (cf. Wehr, A Dictionary of Modern Written Arabic). Steingass (Comprehensive Dictionary, 695) states balsam of the bān tree. 


\begin{tabular}{|l|c|c|c|c|c|c|}
\hline Germander $^{\mathbf{1 4 0}}$ & 7 & $2 \mathrm{~h} / 3 \mathrm{~d}$ & 64 & 7 & 7 & 64 \\
\hline Wild garlic $^{\mathbf{4 1 1}}$ & 7 & $4 \mathrm{~h} / \mathrm{d}$ & 128 & 7 & 7 & 128 \\
\hline Wild carrot $^{\mathbf{1 4 2}}$ & 7 & $3 \mathrm{~h} / \mathrm{d}$ & 34 & 7 & 7 & 34 \\
\hline Melilot $^{\mathbf{1 4 3}}$ & 7 & $1 \mathrm{~h} / \mathrm{d}$ & 16 & 7 & 7 & 16 \\
\hline Gentiana $^{\mathbf{2}}$ & 7 & $3 \mathrm{~h} / 2 \mathrm{~d}$ & 56 & 7 & 7 & 28 \\
\hline Oil of balsam $^{\mathbf{1 4 4}}$ & 7 & $3 \mathrm{~h} / 2 \mathrm{~d}$ & 56 & 7 & 7 & 28 \\
\hline
\end{tabular}

$8 a$

\begin{tabular}{|c|c|c|c|c|c|c|}
\hline Ḳūfiyūn ${ }^{145}$ & 7 & $4 \mathrm{~h} / \mathrm{d}$ & 112 & 7 & 7 & 112 \\
\hline Bdellium $^{146}$ & 7 & $2 \mathrm{~h} / \mathrm{d}$ & 28 & 7 & 7 & 28 \\
\hline Rue $^{147}$ & 2 & $3 \mathrm{~h} / \mathrm{d}$ & 16 & 2 & 2 & 16 \\
\hline Rue seeds & 3 & $3 \mathrm{~h} / \mathrm{d}$ & 24 & 3 & 3 & 24 \\
\hline Greek nard $^{148}$ & 5 & $1 \mathrm{~h} / \mathrm{d}$ & 10 & 5 & 5 & 10 \\
\hline Mastic & 5 & $2 \mathrm{~h} / \mathrm{d}$ & 20 & 5 & 5 & 20 \\
\hline Gum arabic $^{149}$ & 5 & $1 \mathrm{~h} / \mathrm{d}$ & 10 & 5 & 5 & 10 \\
\hline Milk parsley ${ }^{150}$ & 5 & $3 \mathrm{~h} / \mathrm{d}$ & 40 & 5 & 5 & 40 \\
\hline Caraway $^{151}$ & 5 & $3 \mathrm{~h} / \mathrm{d}$ & 40 & 5 & 5 & 40 \\
\hline Opium & 5 & $4 \mathrm{~h} / \mathrm{d}$ & 5 & 80 & 5 & 80 \\
\hline Fennel seeds ${ }^{152}$ & 5 & $2 \mathrm{~h} / \mathrm{d}$ & 20 & 5 & 5 & 20 \\
\hline Red rose & 5 & $1 \mathrm{~h} / \mathrm{d}$ & 10 & 5 & 5 & 10 \\
\hline Hop marjoram ${ }^{153}$ & 5 & $3 \mathrm{~h} / \mathrm{d}$ & 40 & 5 & 5 & 40 \\
\hline Anise $^{154}$ & 3 & $2 \mathrm{~h} / 3 \mathrm{~d}$ & 12 & 3 & 3 & 24 \\
\hline
\end{tabular}

141 Or Chinese cinnamon tree (cf. Wehr, A Dictionary of Modern Written Arabic). Steingass (Comprehensive Dictionary, 695) states balsam of the bān tree.

142 Ibn Sahl, Dispensary, 241.

143 Kindi, Formulary, 251.

144 Kindī, Formulary, 269. The MS states that some copies read: 'seed of balsam'.

145 Kindi, Formulary, 235.

146 Beneath this entry, the MS reads: va-habb balsān fi ba'd al-nuskha.

147 Cf. Ibn Sahl, Dispensary, 175. Oliver Kahl, in the footnotes to the Arabic, suggests that küfiyūn is an Egyptian compound incense.

148 See also Steingass, Comprehensive Dictionary, 1295. Steingass explains that makl could also be a fragrant mixture that includes, aloe, amber, and sandal.

149 Kindi, Formulary, 279-81.

150 Kindi, Formulary, 286-7.

151 Ibn Sahl, Dispensary, 244.

152 Ibn Sahl, Dispensary, 241.

153 Ibn Sahl, Dispensary, 243.

154 Ibn Sahl, Dispensary, 244. 


\begin{tabular}{|l|c|c|c|c|c|c|}
\hline Sweet reed $^{\mathbf{1 5 5}}$ & 3 & $2 \mathrm{~h} / \mathrm{d}$ & 6 & 3 & 3 & 6 \\
\hline Valerian $^{\mathbf{1 5 6}}$ & 3 & $4 \mathrm{~h} / \mathrm{d}$ & 48 & 3 & 3 & 48 \\
\hline Hair $^{\mathbf{1 5 7}}$ & 3 & $3 \mathrm{~h} / \mathrm{d}$ & 24 & 3 & 3 & 24 \\
\hline Sagapenum $^{\mathbf{1 5 8}}$ & 3 & $3 \mathrm{~h} / 2 \mathrm{~d}$ & 24 & 3 & 3 & 12 \\
\hline Asarabacca $^{\mathbf{1 5 9}}$ & 3 & $3 \mathrm{~h} / \mathrm{d}$ & 24 & 3 & 3 & 24 \\
\hline Acacia $^{\mathbf{1 6 0}}$ & 4.5 & $1 \mathrm{c} / 2 \mathrm{~d}$ & 4.5 & 10 & 10 & 4.5 \\
\hline Skink's trunk $^{\mathbf{6 1}}$ & 4.5 & $2 \mathrm{~h} / 1 \mathrm{~d}$ & 10 & 4.5 & 4.5 & 9 \\
\hline (Greek) hypericum $^{\mathbf{1 6 2}}$ & 4.5 & $2 \mathrm{~h} / \mathrm{d}$ & 18 & 4.5 & 4.5 & 18 \\
\hline $\begin{array}{l}\text { Old wine } \\
\mathbf{1 6 3}\end{array}$ & 25 & $2 \mathrm{~h} / \mathrm{d}$ & 100 & 25 & 25 & 100 \\
\hline $\begin{array}{l}\text { Honey equal to thrice } \\
\text { the total }^{-9}\end{array}$ & 996 & $2 \mathrm{~h} / \mathrm{d}$ & 3984 & 996 & 996 & 3984 \\
\hline
\end{tabular}

Therefore, if the hot, cold, moist, and dry parts are combined separately, then we know that the sum of the hot parts is approximately on the measure of four cold parts. And likewise, the dry parts are on the measure of four moist parts. Therefore, we say that Theriac of Mithridates is hot in the second degree. The Şayh also decreed in his treatise on heart medicine: "If I contemplated between myself and my soul, I would find the heat of the theriac at the end of the second degree and the heat of the Mithridates less than it by a little."164

156 Ibn Sahl, Dispensary, 241. See also Steingass, Comprehensive Dictionary, 940.

157 From the Persian mü. Cf. Steingass, Comprehensive Dictionary, 1886-7.

158 Kindi, Formulary, 283-4. Steingass (Comprehensive Dictionary, 680) states that sakbinac is also gum arabic.

159 Kindi, Formulary, 227.

160 Kindi, Formulary, 234.

161 Kindī, Formulary, 245. Steingass (Comprehensive Dictionary, 687) defined sakankūr this as a species of newt or crocodile burying in the sand.

162 Kindi, Formulary, 242.

163 Ibn Sahl, Dispensary, 244.

164 From 'If I contemplated' to the end of the paragraph, Cālīnūs has drawn on Ibn Sīnā's Kitāb al-Adwiya al-qalbiyya in Muḥammad Zuhayr al-Bābā (ed.), Min Mu'allafät Ibn Sīnā al-țibbiyya (Aleppo: Ma'had alturāth al-'ilmī al-'arabī; Cairo: Ma'had al-makhțūțāt al-'arabiyya, 1984): 284. 


\begin{tabular}{|c|c|c|c|c|c|c|}
\hline $\begin{array}{l}\text { The parts of the Lüğgaziya } \\
\text { electuary }^{165}\end{array}$ & $\begin{array}{c}\text { weight in } \\
\text { drams }\end{array}$ & nature & hot parts & cold parts & $\begin{array}{l}\text { moist } \\
\text { parts }\end{array}$ & dry parts \\
\hline Colocynth $^{166}$ & 4.5 & $3 \mathrm{~h} / \mathrm{d}$ & 36 & 4.5 & 4.5 & 36 \\
\hline Sea onion & 4.5 & $2 \mathrm{~h} / \mathrm{d}$ & 36 & 4.5 & 4.5 & 18 \\
\hline Euphorbium & 4.5 & $1 \mathrm{~h} / 2 \mathrm{~d}$ & 9 & 4.5 & 4.5 & 18 \\
\hline Scammony $^{167}$ & 4.5 & $3 \mathrm{~h} / \mathrm{d}$ & 36 & 4.5 & 4.5 & 36 \\
\hline Black Hellebore $^{168}$ & 4.5 & $3 \mathrm{~h} / \mathrm{d}$ & 36 & 4.5 & 4.5 & 36 \\
\hline Gum ammoniac & 4.5 & $3 \mathrm{~h} / 1 \mathrm{~d}$ & 36 & 4.5 & 4.5 & 9 \\
\hline Garlic germander ${ }^{169}$ & 4.5 & $3 \mathrm{~h} / \mathrm{d}$ & 36 & 4.5 & 4.5 & 36 \\
\hline Bindweed $^{170}$ & 3 & $3 \mathrm{~h} / 2 \mathrm{~d}$ & 24 & 3 & 3 & 12 \\
\hline Wall germander ${ }^{171}$ & 3 & $3 \mathrm{~h} / \mathrm{d}$ & 24 & 3 & 3 & 24 \\
\hline Blue Bdellium & 3 & $2 \mathrm{~h} / \mathrm{d}$ & 12 & 3 & 3 & 12 \\
\hline Aloe $^{172}$ & 3 & $2 \mathrm{~h} / \mathrm{d}$ & 12 & 3 & 3 & 12 \\
\hline Thyme $^{173}$ & 2 & $3 \mathrm{~h} / \mathrm{d}$ & 16 & 2 & 2 & 16 \\
\hline Hypericum & 2 & $2 \mathrm{~h} / \mathrm{d}$ & 8 & 2 & 2 & 8 \\
\hline Indian laurel $^{174}$ & 2 & $2 \mathrm{~h} / \mathrm{d}$ & 8 & 2 & 2 & 8 \\
\hline White horehound ${ }^{175}$ & 2 & $3 \mathrm{~h} / 2 \mathrm{~d}$ & 16 & 2 & 2 & 8 \\
\hline Greek spikenard & 2 & $2 \mathrm{~h} / \mathrm{d}$ & 8 & 2 & 2 & 8 \\
\hline Cassia $^{176}$ & 2 & $3 \mathrm{~h} / \mathrm{d}$ & 16 & 2 & 2 & 16 \\
\hline Germander & 2 & $2 \mathrm{~h} / \mathrm{d}$ & 8 & 2 & 2 & 8 \\
\hline White pepper & 2 & $4 \mathrm{~h} / \mathrm{d}$ & 32 & 2 & 2 & 32 \\
\hline Black pepper & 2 & $4 \mathrm{~h} / \mathrm{d}$ & 32 & 2 & 2 & 32 \\
\hline
\end{tabular}

166 Kindi, Formulary, 262.

167 Ibn Sahl, Dispensary, 243. Cf. Steingass, Comprehensive Dictionary, 1190.

168 Kindi, Formulary, 263.

169 Ibn Sahl, Dispensary, 245.

170 Kindi, Formulary, 233-4.

171 Ibn Sahl, Dispensary, 242.

172 Kindi, Formulary, 297.

173 Kindi, Formulary, 256.

174 Ibn Sahl, Dispensary, 244.

175 Ibn Sahl, Dispensary, 241.

176 Ibn Sahl, Dispensary, 244. Steingass (Comprehensive Dictionary, 695) defined saliha as the balsam of the bān tree before it is prepared. 
$9 a$

\begin{tabular}{|l|c|c|c|c|c|c|}
\hline $\begin{array}{l}\text { The rest of the parts of } \\
\text { Lügáziya }\end{array}$ & $\begin{array}{c}\text { weight in } \\
\text { drams }\end{array}$ & nature & hot parts & cold parts & $\begin{array}{c}\text { moist } \\
\text { parts }\end{array}$ & dry parts \\
\hline Long pepper & 2 & $3 \mathrm{~h} / \mathrm{d}$ & 16 & 2 & 2 & 16 \\
\hline Saffron & 2 & $1 \mathrm{~h} / \mathrm{d}$ & 4 & 2 & 2 & 4 \\
\hline Cinnamon & 2 & $3 \mathrm{~h} / \mathrm{d}$ & 16 & 2 & 2 & 16 \\
\hline Opopanax & 2 & $3 \mathrm{~h} / \mathrm{d}$ & 16 & 2 & 2 & 16 \\
\hline Common polypody $\mathbf{1 7 7}^{\mathbf{7 7}}$ & 2 & $3 \mathrm{~h} / 2 \mathrm{~d}$ & 16 & 2 & 2 & 8 \\
\hline Sagapenum & 2 & $3 \mathrm{~h} / 2 \mathrm{~d}$ & 16 & 2 & 2 & 8 \\
\hline Beaver testicle & 3 & $3 \mathrm{~h} / 2 \mathrm{~d}$ & 16 & 2 & 2 & 8 \\
\hline Myrrh & 2 & $2 \mathrm{~h} / \mathrm{d}$ & 8 & 2 & 2 & 8 \\
\hline (Milk) parsley & 2 & $2 \mathrm{~h} / \mathrm{d}$ & 8 & 2 & 2 & 8 \\
\hline Long aristolochia & 2 & $3 \mathrm{~h} / 2 \mathrm{~d}$ & 16 & 2 & 2 & 8 \\
\hline Absynthium & 2 & $1 \mathrm{~h} / 2 \mathrm{~d}$ & 4 & 2 & 2 & 8 \\
\hline Euphorbium & 2 & $4 \mathrm{~h} / \mathrm{d}$ & 32 & 2 & 2 & 32 \\
\hline Indian spikenard & 2 & $1 \mathrm{~h} / 2 \mathrm{~d}$ & 4 & 2 & 2 & 8 \\
\hline Grape ivy ${ }^{\mathbf{1 7 8}}$ & 2 & $2 \mathrm{~h} / \mathrm{d}$ & 8 & 2 & 2 & 8 \\
\hline Ginger & 2 & $3 \mathrm{~h} / 2 \mathrm{~d}$ & 16 & 2 & 2 & 8 \\
\hline Gentiana & 1.5 & $3 \mathrm{~h} / \mathrm{d}$ & 12 & 1.5 & 1.5 & 12 \\
\hline French lavender & 1.5 & $1 \mathrm{~h} / 2 \mathrm{~d}$ & 3 & 1.5 & 1.5 & 6 \\
\hline Honey & 282 & $2 \mathrm{~h} / \mathrm{d}$ & 1128 & 282 & 282 & 1128 \\
\hline The total & $\mathbf{3 7 3}$ & & $\mathbf{1 7 8 0}$ & $\mathbf{3 7 6 . 5}$ & $\mathbf{3 7 6 . 5}$ & $\mathbf{1 6 9 3}$ \\
\hline
\end{tabular}

If it is as it is, then this medicine contains hot parts that are four and a half times the measure of the cold parts. Also,

$9 \mathrm{~b}$

if it increases by $86^{180}$, then this compound is hot at the beginning of the third degree. Likewise, if the dry parts are on the measure of four and half moist parts, then this compound is dry at the beginning of the third degree.

The fourth chapter. Then we say that if you wish to know, with the other degrees' variance from any single degree, what amount of medicine to add so that it shall return to equilibrium, it is necessary to know that if one weight-measure of

177 Kindi, Formulary, 243.

178 Ibn Sahl, Dispensary, 241. Steingass (Comprehensive Dictionary, 430) defined hamāmā as stone parsley or amomum.

179 The sum should be 1695.

180 That is because the cold parts (376.5) times 4.5 plus 86 equal 1780 (really 1780.25). 
our medicine is hot in the fourth [degree] and we wish that with this all of it, being added to a cold medicine, makes it equilibrate. We say that if this cold medicine should be cold in the fourth degree, it is necessary that it be together with this hot medicine so that the compound should be equilibrate. We do not intend, by saying that it is together, that they should be together in weight or quantities. Rather, the intention is thus, that they should be together in their measures. That means that in each weight-unit of medicine, it was demonstrated, in the third preliminary, that they [hot and cold] should be together.

If a cold medicine is cold in the third degree, then it must be one and three-quarters weight-units of medicine so that it might produce an equilibrium for this hot medicine, because in this measure there are at a time hot parts and cold parts, each of which is six and three-quarters parts. For example, it is necessary to mix one weight-unit of pepper and one and three-quarters ${ }^{181}$ weight-units of salsify so that the compound might be at equilibrium. If this cold medicine should be cold in the second degree, it is necessary that it be two weight-units of medicine, because in this measure at any time the cold and hot medicines' parts are seven at a time. For example, if we combine one weight-unit of pepper with two weight-units of cotton of gossamer, the compound is equilibrate because the cold parts are together with the hot parts, for each one is seven at a time. If we combine it with [something] cold in the first, it is necessary that it be four weight-units of cold so that the above-mentioned pepper's single weight-unit returns to equilibrium, because at that time the hot and cold parts are nine. But if you wish to make one weightunit of heat in the third degree together with one weight-unit of cold in the second return to equilibrium, then it is necessary to mix in one and one-half weight units of cold in the second, because at that time the cold and hot parts are five and onehalf. ${ }^{182}$ But if it is from [something] cold in the first, it is necessary to mix three

181 Here, Mũsā Cālīnūs has adopted Ibn Rushd's doctrine of the ratios between degrees. The weight measures of cold medicine necessary to bring to equilibrium a medicine hot in the fourth degree are, approximately on the ratios of 4:3 (with a medicine cold in the third degree), 4:2 (with a medicine cold in the second degree), and 4:1 (with a medicine cold in the first degree). To get the numbers of hot and cold parts for each degree, it is necessary to presume, following Kindi and contra Ibn Rushd, that a medicine hot in a particular degree also had a half part of cold. To account for why the total number of hot and cold parts with a cold medicine in the third degree is 6.75: 1.75 (1.75 weight-units * cold part) +4 ( 1 weight-unit * 4 th degree of hot) +.5 (cold parts in the pepper) +.5 (hot parts in the salsify). When calculating the cold parts present in the medicine cold in the third degree, Mūsā Cālinnūs did not take into account the intensity of the cold in the same way he took into account the intensity of the heat in the medicine hot in the fourth degree.

182 The 1.5 weight units of cold is because the ratio of the third degree to the second is 3:2. The 5.5 hot and cold parts are computed from: 3 (one weight-unit of heat in the third degree) +1.5 (1.5 weight- 
weight-units in order to make this equilibrate. But if we wish to make something hot in the second degree return to equilibrium, it is necessary to mix one weightunit of [something] hot in the second with three weight-units of [something] cold in the first

$10 \mathrm{a}$

so that it makes it equilibrate, because with it at any one time are hot and cold parts, seven at a time. And by this analogy in the rest of the qualities.

The fifth chapter makes its subjects known: A. ${ }^{183}$ Physicians have ordered that warm tumors, at the time of their increase, should be treated with a mixture of restrainers and resolutives so that the material ( $m a \bar{d} d a)$ will be restrained and resolved. And thus one must ask which approach should make it possible for two opposite actions to occur together, because if opposing medicines balance each other, then each one is opposing its opposite. So, each one's effect would therefore not be apparent and, thusly, the compound would be at equilibrium. If one is more powerful, then the powerful's action would be manifest and the weaker's action would not be manifest. Thus, the physicians deemed it conceivable that a compound medicine, if it were a restrainer, can act due to cold; were it a resolutive, it can act due to heat at the same time. It is necessary to know that if the physicians deemed this thing conceivable in the secondary properties, they did not deem it conceivable in the primary properties because they say, for example, that if one dram of camomile is mixed with one dram of rose, then it is a compound at equilibrium in the primary qualities, although it restrains and dissolves. The response is to recognize that the physician Galen gave a response to that, but it is not the true response because the sense resembled the sensations, just as one sense insinuates two opposing things at the same time, because just as we sense that we vomit some cold water and some hot at the same time, and that the eye's pupil and white perceive things together, it is also likewise with this compound. But this comparison is not suitable because on account of how a sensation of this measure is not material, it occurs through it [i.e., matter].

units * cold part) +.5 hot (the heat in the cold medicine) and .5 cold (the cold in the hot medicine). Again, when calculating the cold parts present in the medicine cold in the second degree, Mũsā Câlīnūs did not take into account the intensity of the cold in the same way he took into account the intensity of the heat in the medicine hot in the third degree. 
His Eminence Muhammad Ibn Rushd did not adopt these conditions. He said in the fifth treatise of his Kulliyyāt: As we say that this medicine is equilibrate in the capacity of the first [degree], meaning that it effects in the body a heat that is intermediate between the heat that is in the first degree and the cold that is in it. Likewise, it is desirable that you understand the matter according to the secondary properties so that the dram of camomile, for example, with the dram of rose should produce a restraining and resolution that is intermediate between the resolution of camomile and the restraining of the rose. This neglect occurred, however, with regard to how they did not divide the secondary properties into degrees so that reference might be made from them to that which is at equilibrium and to that which is non-equilibrate. This effect that belongs to the compound medicine is one, whether through an artificial or natural mixture. And it is not long until we need to say how a single medicine can produce two opposite qualities in a single locus (maw $\mathbf{u}_{\bar{u}}$ ). The bodily reactions are like the body's reactions to the medicine,

\section{$10 \mathrm{~b}$}

with it being incorrect that its opposites [can] be found in a single locus at a single time, except with regard to how the intermediate is found between the extremes, as you said, according to the way that white and black are both found in yellow. And if not, they [either] hindered each other necessarily if they were equal or the dominant [one] exerted its effect. ${ }^{184}$

But my opinion is this: This is conceivable accidentally in the secondary properties, just as it occurs also in simple medicines, as Galen explained about absinthe in his A ğlūkānn. ${ }^{185}$ Absinthe has two properties, one constipating and the other laxative ${ }^{186}$, and that this is the same with rose, camomile, colchicum, cabbage, sea-holly, and milk. This same author says that absinthe is a compound of a cold earthy substance and an earthy, fiery, aflame element and cites the following evidence: Its

185 This paraphrase of Ad Glauconem comes from Ibn al-Bayțār (unknown editor), Kitāb al-Jāmi' li-mufradāt al-adwiya wa-'l-aghdhiya, (Baghdād: Maktabat al-Muthannā, 1900-87), vol. 1 of 4:43.

186 On Galen, see the Arabic MS of Ad Glauconem (Risāla ilā Ağlūkān), 46a-b On 47a-b, the Arabic says that it is cold by accident, not by essence. The MS is Wellcome MS Or. 62 (accessed at: https://www.wdl. org/ar/item/15435/view/1/136/). Osler MS 7508 of the Ğāfikī herbal [al-Ghāfiqī (ed. Adam Gacek, Pamela Miller, F. J. Ragep, and Faith Wallis, The Herbal of al- Ghāfiqī (Montreal and Kingston: McGillQueen's University Press, 2014)] lists absinthe on 18b-19a but there is not a strong parallel. For Galen's statement on the composition of absinthe, see Galen: (ed. Karl Gottlob Kühn), Opera Omnia, (Leipzig: C. Cnobloch, 1821-33), vol. 11 of 20: 844. Still, Ibn al-Baytār's Jāmi' is the most likely proximate source because Ibn al-Baytār cited Galen's Risāla ilā Ağlūkān. 
taste is astringent with bitterness and burning. ${ }^{187} \mathrm{He}$ also remarked that coriander is compounded from opposing elements, so it contains a bitter part and watery moisture, as well as a slight seizing that causes it to exert different, varied effects. ${ }^{188}$ For example, it cures low-level erysipelas if taken in a bandage with barley flour and resolves scrofulosis if it is taken in a bandage with bean flour and rose ( $g u l)$, regarding which he said was rose (al-vard). If this medicine is compounded from a cold and light earthy element, a hot airy [element], and a watery [element], we say, after seeing that this is conceivable in a simple medicine, that it is a simple [medicine]. It was unified through the mixture of the natures, because it is conceivable that such a medicine produces opposite effects. Therefore, given that it is possible that there might be, in an artificial compound, a great deal [of opposite effects], we can also say that in the secondary properties it is also conceivable that they can be accidental at different times, that is one following another, and opposite effects. This is why the Şayh says in his treatise not to wash wild chickory/endive. ${ }^{189}$ Colchicum is compounded from two substances, constipating and laxative. If the innate heat and the natural property acted, then the delicate laxative would separate off and its effect would be exerted as resolving and attracting the compound intertwined in the joints until it is free of it. The cold dry substance follows it after a period of time, restrains these members and passageways, grips them, and forces them to prevent the return of what flowed out and to pour out what melted from someplace else into it. Thus it is one of the most useful things for curing joints or that it is an agent, I mean a compound united with an untrue union, or likewise an influence. I mean that it not be a single, true body or organ; it is not necessary that it truly also be a single action and influence.

On account of that, the Şayh also said in the above-mentioned epistle that "camomile has a restraining property and a resolutive property. ${ }^{190}$ If it was used for tumors/abscesses

Ibn al-Baytār, Kitāb al-Jāmi', vol. 1, 42. See also Isaac Israeli [ed. Muḥammad al-Ṣabbāh, Kitāb al-Aghdhiya wa-'l-adwiya, (Beirut: Mu'assasat 'Izz al-Dīn, 1992): 428]. According to Isaac Israeli, absinthe's heat was in the first degree, and its dryness was in the third degree.

188 For Galen's words, see Ibn al-Bayțār, Kitāb al-Jāmi', vol. 4, 66. See also Isaac Israeli (Kitāb al-Aghdhiya, 468).

189 There is a reference to hindibä' birrī in Ibn Sīnā's Kitāb al-Adwiya al-qalbiyya, 272. Ibn Sīnā (Mūjaz, 78) talks about how washing endive makes it weaker.

190 This sentence is also on Ibn Sīnā, Müjaz, 77. 
or given to drink during phlegmy cold fevers, nature, by the permission of its exalted creator, distinguished between the two properties. Therefore, I have called upon the cold for help in extinguishing the heat that overcomes the members, and upon the heat for help in resolving the frozen, cold substance in fevers. In the case of tumors, it directs the cold property to the passages and the apertures, narrows them, and prevents the material from reaching them and the material directed toward the organ. When it occurs in it a little later it restrains it, so it is wary of it and washes it out. It hardens it and prevents it from flowing as it used to the organ's substance (cevher), so it pleases it and strengthens it and it is not influenced by the odious matter. It directs the hot property to the nearby matter in the organ so that it dissolves that matter and cleans it out."191

The second question is this: The third premise was not truthful in its explanation because apparently we see that the effect of a certain rank's medicines together is not from their measures together. Thus Avenzoar mentioned their measure in the epistle that he made for the Padişah ${ }^{192}$ so that every single medicine's rank would be clear.

The third question is this: The fourth premise was not true, just as Muhammad Ibn Rushd, in the fifth book of his Kulliyyāt, explained saying: The man known as al-Kindi made ${ }^{193}$ the ratio of the four degrees of the degrees of medicines to be the ratio of the doubles, so that the fourth degree is sixteen times [the first]. This is because he made the first twice equilibrium, the second twice the first, the third twice the second, and the fourth twice the third. Is it not enough for him to say that the second is twice the first, that the third three times it, and that the fourth four times it? That is what he intended in ranking them, so that their ranks would be equal.

191 This entire quotation is from Ibn al-Bayțār, al-Jämi', vol. 1, 74. Although the phrase 'above-mentioned epistle' might refer to the treatise on heart medicines, I could not find this quoted passage in the printed edition of Kitāb al-Adwiya al-qalbiyya. Just before the quoted portion, Ibn al-Baytāa reported (al-Jāmi', vol. 1, 74): qāla al-shaykh al-ra'îs fì maqālatih fï al-hindibä'. Thus, it is more likely a reference to Ibn Sīnā's Treatise on Hindibä' which exists in MSS.

192 Ibn Rushd may have referenced this text by Ibn Zuhr in Kitāb al-tiryāq, found in Ibn Rushd (Jūrj Qanawātī and Sa'īd Zā'id eds.), Rasā'il Ibn Rushd al-țibbiyya, (Cairo: al-Hay'a al-Mi riyya al-'āmma li'l-kitāb, 1987): 387-422, at 397; see also 418. See also Lucien Leclerc, Histoire de la Médecine Arabe, (Paris: Ernst Leroux, 1876), vol. 2: 87. The research of Melitta Adamson ["Bernard de Gordon and Arnald de Villanova: A Tale of Two Regimes," in Andreas Speer and David Wirmer (eds.), 1308: Eine Topogaphie historischer Gleichzetigkeit, (Berlin/New York: Walter de Gruyter, 2010): 419-35 at 424-5] has found that Arnaldo could refer to Ibn Zuhr's treatises without naming a title. Thus, it is possible that Mūsā Cālinnūs' knowledge of Ibn Zuhr's work came, entirely or partially, from Arnaldo.

This third question starts on Ibn Rushd, Kulliyyāt, 391 (five lines up from the bottom). 
That is, they took the first medicine from which there appears in the body a sensible heat and placed it in the first degree. Then they turned to a medicine whose distance from it is the same distance of the first from equilibrium, and placed it in the second degree. There is no doubt that this is twice the first. Then they turned to a medicine whose distance from the second is the same distance of the second from the first, and placed it in the third. In this are three times the first and likewise in the fourth. According to al-Kindi's opinion, it is necessary that they would have made the second increase over the first by doubling the increase of the first over the mean, and the third over the second by twice

\section{$11 b$}

what the second increases over the first. I wish I knew what necessity induced the physicians to retain this ratio. Likewise, the medicines that were in the third degree would have exceeded the fourth and thus become lethal, what would the state of bodies be after being treated with medicines that exceed equilibrium by sixteen (times)? ${ }^{194}$ Also, the distance of the fourth degree from the third would not have been the same as the distance of the third from the second, but rather the distance of the third from the mean. It was incumbent upon them, in the likes of this objective, that they separate by degrees, and likewise with what is between the third and the second. Thus the ranks of the degrees are not equal, and which disorder in this craft is greater than this one? So, what he [Kindi] intended for it, from the very beginning, with regard to the preservation ${ }^{195}$ of the ranks of some properties' increase over others eludes us. Namely, for example, the rank whose ratio to the first is equivalent to the ratio of the first to equilibrium eludes us and, more than that, what was between the other degrees, because according to al-Kindi, whenever the magnitude of the width between them increased until, were there a fifth degree, there would be 32 parts, because they would increase over the fourth [degree] by sixteen parts. All of this is ravings and superstitions, for he spoke about things that lack any existence. One aspect of al-Kindì's error is that he made that which is in the first degree to be double the hot and cold qualities at equilibrium, and it was necessary for him to follow the ratio of doubling.

One might say that what the physicians meant by the first degree was what is increased over equilibrium by one part from sixteen. Accordingly, if the ratio of 
doubling were compounded by the increase of the degrees, it is not necessary that the medicine in the fourth degree be sixteen times that which is at equilibrium. One might thus respond that Galen said that I mean by the first degree what appears to the senses as soon as it appears from the changes of the body, and were the desired [meaning] of the first degree to be twice equilibrium, the alteration of the body that appears in the body from it [the medicine] would not be the first alteration. Consider this, and it is clear. But the habit of the people is, if a famous man erred, to follow him due to how blind imitation overcomes their dispositions.

The fourth question is that this might be said to the fifth premise, I mean the rule that

$12 \mathrm{a}$

in the first premise: A medicine with a lesser degree should cause the degree of a greater degree to descend. For example, if something at a lesser heat is mixed with something that has a greater heat, then there is a decrease in the heat of the greater heat. For example, if tepid water is mixed with strongly bubbling water, then we say that there is a decrease in its heat. If this rule is true, then it is necessary for very hot diseases, for example, those hot in the third or fourth degrees. Take the case of a burning fever. If we give something hot in the first or second degrees, it is necessary that it, being beneficial to its fever, be able to diminish it. We see that it is extremely harmful to it [the burning fever], but here it should not be harmful. We see some cold moist things, for example, the juice of cucumbers and fruits that wither, as harmful, especially that something hot is necessarily extremely harmful. But because of this answer, we say that with something having been mixed, this thing may truly decrease in actuality or potentially. But if one of them shall be in potential and the other one in actuality, then it is conceivable that this rule is deceptive because if the above-mentioned feverish thing will give heat in the first [degree] or cold in the first, with this medicine also currently remaining in the body, perhaps it would have been beneficial. But with the innate and accidental heat burning it and returning it to a putrid yellow through conflagration, it is harmful. Things that are a little hot are also harmful accidentally and in more than one way because they increase the heat accidentally, just as ironworkers sprinkle a little water on a fire so that it is said to be more powerful because every contrary opposing its opposite comes to strengthen it. Because of this, very little cold water should not be given to those with burning fevers or during epidemics. Much is given to drink all at once, impediments being absent. 
The fifth question is also about the fifth preliminary. We say that it is inconceivable that something with a lower degree should be able to cause something with a higher degree to decrease by this amount, just Muhammad Ibn Rushd explained ${ }^{196}$ by saying that when there are medicines of opposing properties, some of the properties reduce others by the measure of the equilibrium of the opposition that is in them. I mean, for example, that the medicine that is cold in the first [degree] reduces the second in heat by the amount that the cold has increased in it, it being a single degree. It is also necessary with medicines of homogeneous properties that the weakest property of them reduces the strongest

$12 \mathrm{~b}$

according to the measure of the ratio of the opposite in the weaker medicine to its opposite in the stronger medicine by the greatest ratio it has to the strongest medicine. An example of this is that with something hot in the first degree, its cold's greatest ratio to the heat in the medicine that is hot in the second and third degrees is smaller than it is in the second. If this is so, the equilibrate of the medicines with homogeneous properties is the nearest of the ranks in that it reduces that which is above it if the ratio of the opposite in it approaches the ratio of equilibrium. Then, after it, what was in the first degree, then in the second degree, then in the third degree.

An example of this is when we mix an equilibrate medicine with something hot in the second degree, it cannot direct it to the first degree because that which does so is something cold in the first [degree]. But it reduces it by an amount so that it does not reach this rank. If we mix something hot in the first with it, instead of the equilibrate, it also reduces it, but less than that by which the equilibrate reduces it if the hot medicine were in the first [degree]. The ratio of the cold in it to the hot is smaller than its ratio to it in the equilibrate, just as the ratio in the equilibrate is smaller than its ratio in the [medicine] that is cold in the first. Thus the equilibrate medicine could not have reduced the hot in the second, as much as it reduces the hot in the first. It is also not possible for something hot in the first [degree] to reduce something hot in the second as much as the equilibrate would. It is also not possible for [something] hot in the second degree to reduce something hot in the third degree like something hot in the first would reduce [it] or, for that matter, like the equilibrate or the cold in the first would. But all of this is after it has decreased 
through the equivalence of the quantities, I mean the equivalence of the properties. All of this, if it is contemplated, is clear on its own. More recent physicians are ignorant of these things. You see them saying that if the medicine that is hot in the first were mixed with something hot in the third, then it would make it into something hot in the second. I wish I knew, for if we mixed it with something cold in the first to any degree, it would make it into something cold. If they said to the second rank, then the hot in the first degree and the cold in the first degree would come to make the heat in the third on one rank. If they said that the cold in the first makes the heat in the third degree into heat in the first degree, then it makes the cold in the second degree into heat in the third degree, meaning that it is equilibrate. All of this is grasping about. And God is most knowing.

\section{Conclusions}

This transcription and annotated translation of Risāla fi țaba’’ ${ }^{\prime}$ al-adviya va-isti'mālihā has made the following contributions. First, this article is the first publication devoted to this treatise by Mūsā Cālinnūs, a figure whose Arabic and Hebrew writings have already attracted some scholarly attention. Second, the present article's introduction noted that there was not much scientific literature in Ottoman Turkish that predated this treatise. Thus, it is an important source for the development of Ottoman Turkish scientific literature during the early sixteenth-century Ottoman Empire. Third, and above all, this text is important evidence that the court of Bayazid II was interested in Latin medical texts and for how information from those texts made it to the Ottoman Empire. While scholars have known of contacts between Europeans and the Ottoman court in the late-fifteenth and early-sixteenth centuries, Risāla fi țabä’’ al-adviya provides important details about the effect of those contacts on Ottoman intellectual life.

\section{Bibliography}

Adamson, Melitta. "Bernard de Gordon and Arnald de Villanova: A Tale of Two Regimes." In 1308: Eine Topographie historischer Gleichzeitigkeit. Andreas Speer and David Wirmer eds. Berlin and New York: Walter de Gruyter, 2010: 419-35.

Adamson, Peter. al-Kindī. Oxford and New York: Oxford University Press, 2006.

Adnan-Adıvar, Abdülhak. Osmanlı Türklerinde İlim. Istanbul: Maarif Matbaası, 1943.

al-Ghāfiqī (ed. Adam Gacek, Pamela Miller, F. J. Ragep, and Faith Wallis). The Herbal of al-Ghäfiqī. Montreal and Kingston: McGill-Queen's University Press, 2014.

al-Kindī: The Medical Formulary or Aqrābādhīn of al-Kindī. Translated with a Study of its Materia Medica by Martin Levey. Madison, Milwaukee, and London: The University of Wisconsin Press, 1966. 
al-Ṭabarī, Alī b. Sahl (ed. M. Z. Siddiqi). Firdaws al-ḥikma fi al-țibb. Berlin: The E.G.W. Gibb Memorial Trust and Sonnen-Druckerei, 1928.

Arnaldo de Villanova (ed., intro., and comm. Michael R. McVaugh). Opera medica omnia. 2, Aphorismi de gradibus. Granada and Barcelona: 1975.

Beinart, Haim. "Lorki (i.e., of Lorca), Joshua." Encyclopaedia Judaica. Ed. Michael Berenbaum and Fred Skolnik. 2nd ed. Vol. 13. Detroit: Macmillan Reference USA, 2007. 192-193. Gale Virtual Reference Library. Web. 4 Sept. 2016.

Chipman, Leigh. The World of Pharmacy and Pharmacists in Mamlük Cairo. Boston: Brill, 2010.

Demaitre, Luke E. Doctor Bernard de Gordon: Professor and Practitioner. Toronto: Pontifical Institute of Mediaeval Studies, 1980.

Duran, Recep. “Nefsü'l-Emr Risaleleri.” Ankara Üniversitesi Dil ve Tarih-Coğrafya Fakültesi Araştırma Dergisi XIV (1992): 97-106.

Fancy, Nahyan. "Medical Commentaries: A Preliminary Examination of Ibn al-Nafīs's Shurūḥ, the Müjaz, and Subsequent Commentaries on the Müjaz." Oriens XLI (2013): 525-45.

---. Science and Religion in Mamluk Egypt: Ibn al-Nafis, pulmonary transit and bodily resurrection. London: Routledge, 2013.

Fazlığlu, İhsan. "Between Reality and Mentality- Fifteenth Century Mathematics and Natural Philosophy Reconsidered.” Nazariyat I (2014): 1-39.

Galen (trans. P.N. Singer). Selected Works. Oxford and New York: Oxford University Press, 1997.

Galen: (ed. Karl Gottlob Kühn): Opera omnia. Leipzig: C. Cnobloch, 11:1821-33.

Gauthier, Léon. Antécédents gréco-arabes de la psychophysique. Beirut: Imprimerie Catholique, 1938.

Günergün, Feza. "Ottoman Encounters with European science: sixteenth and seventeenth-century translations into Turkish." In Cultural Translation in Early Modern Europe. P. Burke and R. Po-chia Hsia eds. Cambridge: Cambridge University Press, 2007: 192-211.

Huguet-Termes, Teresa. "Islamic Pharmacology and Pharmacy in the Latin West: An Approach to Early Pharmacopeias." European Review 16, no. 2 (2008): 229-39.

Ibn al-Bayțār (unknown editor). Kitāb al-Jāmi' li-mufradāt al-adwiya wa-'l-aghdhiya. Baghdād: Maktabat al-Muthannā, 1:1900-87.

Ibn al-Nafīs (ed. 'Abd al-Karīm al-'Azabāwī). al-Mūjaz fi al-țibb. Cairo: Wizārat al-awqāf, 1986.

Ibn al-Tilmīdh, Hibat Allāh (ed., trans., intro. and comm. Oliver Kahl). The Dispensatory of Ibn at-Tilmīd. Leiden and Boston: Brill, 2007.

Ibn Nahmias, Joseph (ed., trans. and comm. Robert Morrison). The Light of the World: Astronomy in al-Andalus. Berkeley and London: The University of California Press, 2016.

Ibn Rushd (ed. J.M. Fórneas Besteiro and C. Alvarez de Morales). Kitāb al-Kulliyāt fï al-țibb. Madrid: Consejo Superior de Investigaciones Científicas and Granada: Escuela de Estudios Arabes, 1987.

--- (ed., Jūrj Qanawātī and Sa'īd Zā’id). Rasā'il Ibn Rushd al-țibbiyya. Cairo: al-Hay’a al-Mișriyya al-āmma li-'l-kitāb, 1987.

Ibn Sahl, Sābūr. (ed., trans., and comm. Oliver Kahl). Sābūr ibn Sahl's Dispensary in the Recensions of the 'Adudì Hospital. Boston: Brill, 2008.

Ibn Sīnā (ed. Muḥammad Zuhayr al-Bābā). Kitāb al-Adviya al-qalbiyya. Min Mu'allafāt Ibn Sīnā al-țibbiyya. Aleppo: Ma'had al-turāth al-'ilmī al-'arabī and Cairo: Ma'had al-makhțūțāt al-'arabiyya, 1984.

İhsanoğlu, Ekmeleddin (ed.). Osmanlı Tıbbi Bilimler Literatürü Tarihi. Istanbul: Ircica, 2008.

Israeli, Isaac (ed. Muḥammad al-Ṣabbāḥ). Kitāb al-Aghdhiya wa-'l-adwiya. Beirut: Mu’assasat 'Izz al-Dīn, 1992.

İzgi, Cevat. Osmanlı Medreselerinde İlim. Istanbul: İz Yayıncılık, 1997.

Jacquart, Danielle. “Bernard de Gordon et l'astrologie.” Centaurus 45 (2003): 151-58. 
Jacquart, Danielle. "Islamic Pharmacology in the Middle Ages: Theories and Substances." European Review 16, no. 2 (2008): 219-27.

Küçük, B. Harun. "New Medicine and the Hikmet-i țabīityye Problematic in Eighteenth-Century Istanbul." In Texts in Transit. Ed. Y. Tzvi Langermann and Robert G. Morrison. University Park, PA: The Pennsylvania State University Press, 2016: 222-42.

Langermann, Y. Tzvi. “A Compendium of Renaissance Science: Ta'alumot ḥokmah by Moshe Galeano.” Aleph: Historical Studies in Science and Judaism 7 (2007): 283-318.

---. "Another Andalusian Revolt? Ibn Rushd's Critique of al-Kindī's Pharmacological Computus." In The Enterprise of Science in Islam: New Perspectives. Ed. Jan P. Hogendijk and A.I. Sabra. Cambridge and London: MIT Press, 2003: 351-372.

---. "Medicine, Mechanics and Magic from Moses ben Judah Galeano's Ta'alumot Họma." Aleph: Historical Studies in Science and Judaism 9 (2009): 353-77.

Leclerc, Lucien. Histoire de la Médecine Arabe. Paris: Ernst Leroux, 1876.

Levey, Martin. Early Arabic Pharmacology. Leiden: Brill, 1973.

Lorci, Joshua. Gerem ha-ma'alot. Austrian National Library Hebrew 45.

McVaugh, Michael R. "Quantified Medical Theory and Practice at Fourteenth-Century Montpellier." Bulletin of the History of Medicine 43 (1969): 397-413.

Morrison, Robert. “A Scholarly Intermediary Between the Ottoman Empire and Renaissance Europe." Isis 105 (2014): 32-57.

---. "An Astronomical Treatise by Mūsā Cālīnūs alias Moses Galeano." Aleph: Historical Studies in Science and Judaism 10, no. 2 (2011): 315-53.

---. "The Role of Oral Transmission for Astronomy among Romaniot Jews." In Texts in Transit. Ed. Y. Tzvi Langermann and Robert Morrison. University Park, PA: Pennsylvania State University Press, 2016: 10-28.

Nutton, Vivian. “Galen of Pergamum.” Brill's New Pauly. Antiquity volumes edited by Hubert Cancik and Helmuth Schneider. Brill Online: 2016.

Pfeiffer, Judith. “Teaching the Learned: Jalāl al-Dīn al-Dawānī's Ijāza to Mu'ayyadzāda 'Abd al-Raḥmān Efendi and the Circulation of Knowledge between Fārs and the Ottoman Empire at the Turn of the Sixteenth Century." In The Heritage of Arabo-Islamic Learning. Ed. Maurice Pomerantz and Aram Shahin. Leiden and Boston: Brill, 2016: 284-332.

Ragep, Sally. Mạ̣mūd ibn Muḥammad ibn 'Umar al-Jaghminnī’ al-Mulakhkhaṣ fĩ al-hay’a al-basīṭa: An Edition, Translation, and Study. Ph.D. Dissertation, McGill University, 2014.

Samsó, Julio. “Abraham Zacut and José Vizinho's Almanach Perpetuum in Arabic (16th-19th C.)." Centaurus 46 (2004): 82-97.

Savage-Smith, Emilie. "Medicine in Medieval Islam." In The Cambridge History of Science. Ed. Michael H. Shank and David C. Lindberg. Cambridge and New York: Cambridge University Press, 2013, 2: 139-67.

Totelin, Laurence. "Mithradates' Antidote: A Pharmacological Ghost." Early Science and Medicine IX (2004): 1-19. 